\title{
FUNCTIONALS OF $r$-DIMENSIONAL MANIFOLDS ADMITTING CONTINUOUS GROUPS OF POINT TRANSFORMATIONS*
}

\author{
ARISTOTLE D. MICHAL $\dagger$
}

1. Introduction. The study of integral invariants was initiated by $\mathrm{H}$. Poincaré in connection with some important problems in dynamics in his memorable prize memoir $\ddagger$ on the three-body problem. S. Lie§ showed that the subject of integral invariants is closely connected with his theories of differential invariants of continuous groups of transformations. More recently Goursat and Cartan in taking up the subject from Poincaré's point of view have made important contributions; and in their respective books Legons sur le Problème de Pfaff and Leçons sur les Invariants Intégraux they have connected their results with the Pfaffian problem and its generalizations.

A multiple integral extended over an $r$-dimensional manifold in $n$ dimensions depends for its value on the $r$-dimensional manifold and its sense, $\|$ and in general on the parametric representation of the manifold. If one fixes the sense of the manifold and if the multiple integral remains invariant $T$ in value for an arbitrary change of parameters, then the multiple integral will depend only on the $r$-dimensional manifold for its value. In other words, such a multiple integral is a functional of $r$-dimensional manifolds; and hence Poincaré's and Cartan's integral invariants are additive functional invariants. Taking cognizance of this point of view, one naturally inquires as to the

* Presented to the Society under a somewhat different title on January 1, 1926, except the results of $\$ 2$ which were presented to the Society on March 1, 1924. This paper was received by the editors in September, 1926.

$\dagger$ National Research Fellow in Mathematics.

$\ddagger$ Acta Mathematica, vol. 12 (1890). For a more complete exposition see his Méthodes Nouvelles de la Mécanique Céleste, vol. III.

$\S$ Die Theorie der Integralinvarianten ist ein Corollar der Theorie der Differentialinvarianten, Berichte der Sächsischen Gesellschaft der Wissenschaften, 1897, pp. 342-357.

\| Cf. Philip Franklin, Multiple integrals in n-space, Annals of Mathematics, (2), vol. 24 (19221923), pp. 213-226. See also Georges Giraud, Le problème de Dirichlet généralisé, Annales de l'École Normale Supérieure, January, 1926.

I We note here that some of Lie's work on integral invariants (especially with respect to infinite continuous groups) has reference to such an invariance. Such types of integral invariants are also found in works on tensor analysis. Cf. Weitzenböck, Invariantentheorie, chapter XIV; and E. Noether, Göttinger Nachrichten, 1918. 
possibility of considering invariant functionals which are not necessarily additive. This paper is concerned with such generalizations of the Poincare and Cartan integral invariant.

In the first part of the paper we consider first functionals of closed plane curves that have a normal variation and that admit a one-parameter group of the plane. It is found that such invariant functionals are solutions of functional equations with functional derivatives. To get explicit solutions of our problems we assume our functionals to be expansible in a series each term of which is a repeated area integral. It is easy to extend this reasoning to the case of corresponding expansions of functionals of closed $(n-1)$ dimensional manifolds in $n$ dimensions; but the case of functionals of closed space curves requires special considerations. The problem reduces to the solution of a functional equation with functional fluxes. I then indicate briefly what type of functional expansions could be used to solve the equations.

In the second part of the paper certain tensors that depend on $i$ points in space are discussed. Then we consider the invariant theory, under oneparameter groups, of functionals $I_{i}$ having the form of an $i$-tuply repeated $r$-dimensional integral* (see expression (5.4)). In this part of the paper we do not restrict the invariance with respect to closed manifolds only, exception being taken of $\S 11$. In generalizing Goursat's notion of integral invariants attached to the trajectories of a system of differential equations, we are led to a generalization of Cartan's complete integral invariant. One of the most interesting theorems of the paper is that of $\S 12$, which shows the impossibility in general of stepping from a generalized Poincare integral invariant to a generalized Cartan complete integral invariant.

At the end of the paper certain results are given on functional invariants of $s$-parameter groups. I propose to make a more complete study of functional invariants of $s$-parameter groups elsewhere.

There are a number of problems that one may well consider in studying the functional invariants of this paper. For example, the generalization of the Pfaffian problem by means of non-additive functionals offers such a possibility. It is my intention to consider such problems in other papers.

Throughout the whole paper we shall assume without statement, or with slight statement, the necessary continuity, differentiability, analyticity and

\footnotetext{
* It is only a matter of calculation to show that the invariant theory of the functional $\sum_{i=0}^{\infty} I_{i}$ is merely the invariant theory of the representative term. This can be shown by considering an obvious generalization of the lemma of $\$ 2$. For the sake of brevity we make our calculations only for a representative term $I_{\boldsymbol{i}}$.
} 
uniformity properties of our functions in a certain finite region $\Delta$ in which we shall work.

\section{PART I}

2. Invariant functionals of closed plane curves. ${ }^{*} \mathrm{We}$ consider a oneparameter continuous group of transformations defined by the system of differential equations

$$
\frac{d x}{\xi(M)}=\frac{d y}{\eta(M)}=d \tau .
$$

In (2.1) $M$ denotes a point in the plane and $\tau$ denotes the parameter of the group. We shall assume without any further statement that the functions $\xi$ and $\eta$ are real analytic functions of $x$ and $y$ in a certain domain $\Delta$. We further assume that $\xi$ and $\eta$ remain finite in $\Delta$ and do not vanish simultaneously in $\Delta$.

Let $\Phi[C]$ be a continuous functional of closed plane curves possessing a normal functional derivative $\Phi_{n}^{\prime}[C / M]$, taken at a point $M$ on $C$, uniformly continuous in $C$ and $M$. We assume in this paragraph that we are dealing with such functionals $\Phi[C]$ which have a variation of the form

$$
\delta \Phi[C]=\int_{C} \Phi_{n}^{\prime}[C / M] \delta n(M) d s .
$$

(In (2.2) $\delta n(M)$ stands for the variation of the normal to $C$ at the point $M$ on $C$ and $d s$ is the element of arc length of $C$. We shall make the convention that the outer direction of the normal is positive.

We proceed to prove a lemma which we shall find useful in our subsequent discussion.

LEMma. If $F[C]$ is a functional of closed curves possessing a development

$$
F[C]=f^{(0)}+\sum_{i=1}^{\infty} \frac{1}{j !} \int_{\sigma} \int_{\sigma(i)} \cdots \int_{\sigma} f^{(i)}\left(M_{1}, M_{2}, \cdots, M_{i}\right) d \sigma_{1} d \sigma_{2} \cdots d \sigma_{i}
$$

( $\sigma$ is the area within $C$ and $d \sigma_{h}$ is the element of area at a point $M_{h}$ ) with

$$
\left|f^{(i)}\left(M_{1}, M_{2}, \cdots, M_{i}\right)\right|<K
$$

* The analysis given in this paragraph can be extended easily to the case of corresponding invariant problems of functionals of closed $(n-1)$-dimensional manifolds in $n$ dimensions. For an abstract of most of the results of $\$ 2$ see the Proceedings of the National Academy of Sciences, vol. 11 (1925), pp. 98-101. 
and $f^{(i)}\left(M_{1}, M_{2}, \cdots, M_{i}\right)$ symmetric ${ }^{*}$ and continuous in all its $i$ arguments $M_{1}$ to $M_{i}$, then a necessary and sufficient condition that

$$
F[C]=0
$$

for all closed curves is that

$$
f^{(0)}=0, \quad f^{(i)}\left(M_{1}, M_{2}, \cdots, M_{i}\right)=0 \quad(i=1,2,3, \cdots) .
$$

Since the normal functional derivatives of all orders of (2.3) exist and are continuous, it follows from the hypothesis (2.4) that all the functional derivatives of $F[C]$ must vanish. Letting $\sigma$ shrink indefinitely, we get as a necessary consequence

$$
f^{(0)}=0 \text {. }
$$

Taking the first functional derivative of (2.4) at the point $M_{1}$, and letting $\sigma$ shrink indefinitely we get

$$
f^{(1)}\left(M_{1}\right) \equiv 0 \text {. }
$$

On following such a process of functional differentiations followed by letting $\sigma$ shrink indefinitely, we find

$$
f^{(i)}\left(M_{1}, M_{2}, \cdots, M_{i}\right) \equiv 0 \quad(i=1,2,3, \cdots) .
$$

A necessary and sufficient condition that a functional $\Phi[C]$ admit the group (2.1) is that its variation vanish identically in virtue of (2.1). Since along the path curves of (2.1) we have

$$
\delta n(M)=[\xi(M) \cos (x, n)+\eta(M) \cos (y, n)] d \tau,
$$

this condition becomes

$$
\int_{C} \Phi_{n}^{\prime}[C / M][\xi(M) \cos (x, n)+\eta(M) \cos (y, n)] d s=0
$$

for all closed curves $C$.

We can write equation (2.7) in the equivalent form

$$
\Phi_{n}{ }^{\prime}[C / M]=A[C / M]
$$

where $A[C / M]$ is a given arbitrary functional satisfying the functional equation

$$
\int_{C} A[C / M][\xi(M) \cos (x, n)+\eta(M) \cos (y, n)] d s \equiv 0
$$

* This is no essential restriction, as by a process of symmetrization we can put the functional in such a form. 
The integrability conditions* for the functional equation (2.8) can be stated in terms of the notion of an adjoint functional as follows.

A necessary and sufficient condition that (2.8) be integrable is that $\delta A[C / M]$ be a self-adjoint functional of $\delta n$. We may proceed now and show that there always exists an infinitude of functionals $A[C / M]$ that satisfy (2.9) and satisfy the integrability conditions for (2.8) identically. Hence there would always be solutions for a Cauchy problem for (2.8). Instead of following such a general argument, however, we shall assume an explicit expansion for $\Phi[C]$ and impose the conditions that such a $\Phi[C]$ satisfy the equation (2.7).

Assume then that we are considering functionals $\Phi[C]$ developable in an expansion

$$
\phi^{(0)}+\sum_{i=1}^{\infty} \frac{1}{i !} \int_{\sigma} \int_{\sigma} \cdots \int_{(i)} \phi^{(i)}\left(M_{1}, M_{2}, \cdots, M_{i}\right) d \sigma_{1} d \sigma_{2} \cdots d \sigma_{i}
$$

where $\phi^{(i)}\left(M_{1}, M_{2}, \cdots, M_{i}\right)$ is symmetric in all the $i$ points $M_{1}$ to $M_{i}$ and possesses continuous first partial derivatives, and

$$
\left|\phi^{(i)}\right|<K \text {. }
$$

We shall demonstrate the following theorem.

THEOREM 1. A necessary and sufficient condition that a functional $\Phi[C]$ possessing a development (2.10) admit the group of transformations defined by (2.1) is that each $\phi^{(i)}\left(M_{1}, \cdots, M_{i}\right)$ satisfy the corresponding partial differential equation

$$
\begin{array}{r}
\sum_{i=1}^{i}\left\{\xi\left(M_{i}\right) \frac{\partial \phi^{(i)}\left(M_{1}, \cdots, M_{i}\right)}{\partial x_{i}}+\eta\left(M_{i}\right) \frac{\partial \phi^{(i)}\left(M_{1}, \cdots, M_{i}\right)}{\partial y_{i}}\right. \\
\left.+\phi^{(i)}\left(M_{1}, \cdots, M_{i}\right)\left[\frac{\partial \xi\left(M_{i}\right)}{\partial x_{i}}+\frac{\partial \eta\left(M_{i}\right)}{\partial y_{j}}\right]\right\}=0 .
\end{array}
$$

To prove this theorem we make use of the evident relations

$$
\begin{aligned}
& \int_{C} u(M) \cos (x, n) d s=\int_{\sigma} \frac{\partial u}{\partial x} d \sigma, \\
& \int_{C} v(M) \cos (y, n) d s=\int_{\sigma} \frac{\partial v}{\partial y} d \sigma .
\end{aligned}
$$

* Cf. G. C. Evans, The Cambridge Colloquium, pp. 20-23. 
By a direct calculation* we have

$$
\Phi_{n}^{\prime}[C / M]=\phi^{(1)}(M)
$$

$$
+\sum_{i=1}^{\infty} \frac{1}{i !} \int_{\sigma} \int_{\sigma(i)} \cdots \int_{0} \phi^{(i+1)}\left(M, M_{1}, \cdots, M_{i}\right) d \sigma_{1} d \sigma_{2} \cdots d \sigma_{i}
$$

Hence condition (2.7) becomes

$$
\begin{aligned}
& \sum_{i=0}^{\infty} \frac{1}{i !} \int_{\sigma} \int_{\sigma} \ldots \int_{\sigma}\left[\frac{\partial \phi^{(i+1)}\left(M, M_{1}, \cdots, M_{i}\right) \xi(M)}{\partial x}\right. \\
& \left.\quad+\frac{\partial \phi^{(i+1)}\left(M, M_{1}, \cdots, M_{i}\right) \eta(M)}{\partial y}\right] d \sigma d \sigma_{1} d \sigma_{2} \cdots d \sigma_{i}=0
\end{aligned}
$$

for all closed curves $C$.

On symmetrizing the integrands without changing the values of the integrations and on renaming the subscripts, condition (2.14) becomes

$$
\begin{array}{r}
\sum_{i=1}^{\infty} \frac{1}{i !} \int_{\sigma} \int_{\sigma(i)} \ldots \int_{\sigma}\left[\sum _ { j = 1 } ^ { i } \left\{\frac{\partial \xi\left(M_{j}\right) \phi^{(i)}\left(M_{1}, \cdots, M_{i}\right)}{\partial x_{j}}\right.\right. \\
\left.\left.\quad+\frac{\partial \eta\left(M_{j}\right) \phi^{(i)}\left(M_{1}, \cdots, M_{i}\right)}{\partial y_{j}}\right\}\right] d \sigma_{1} \cdots d \sigma_{i}=0 .
\end{array}
$$

Hence by our lemma, a necessary and sufficient condition that (2.15) hold is that $\dagger$

$$
\begin{array}{r}
\sum_{i=1}^{i}\left[\frac{\partial \xi\left(M_{i}\right) \phi^{(i)}\left(M_{1}, \cdots, M_{i}\right)}{\partial x_{j}}+\frac{\partial \eta\left(M_{j}\right) \phi^{(i)}\left(M_{1}, \cdots, M_{i}\right)}{\partial y_{i}}\right]=0 \\
(i=1,2, \cdots) .
\end{array}
$$

Our theorem therefore follows readily.

From the form of the equations (2.11) it is clear that if $\psi\left(M_{1}, \cdots, M_{i}\right)$ is a solution then $\psi\left(M_{l}, \cdots, M_{r}\right)$ is also a solution, where $M_{l}, \cdots, M_{r}$ is any permutation of $M_{1}, \cdots, M_{i}$. Hence there always exist symmetric functions of $M_{1}, \cdots, M_{i}$ that satisfy equation (2.11). Thus there always exist functionals of closed plane curves of form (2.10) admitting a given arbitrary continuous one-parameter group of transformations (2.1).

* Functional differentiations and integrations term by term are valid since the series involved are uniformlyt convergent.

$\dagger$ We note hat this condition affirms the invariance of each term of (2.10). 
We shall prove the following theorem which will give information as to the most general functional invariant of type (2.10).

Theorem 2. If

(2.17) $\Phi[C]=\phi_{0}^{(0)}+\sum_{i=1}^{\infty} \frac{1}{i !} \int_{0} \int_{\sigma} \ldots \int_{0} \phi_{0}^{(i)}\left(M_{1}, \cdots, M_{i}\right) d \sigma_{1} \cdots d \sigma_{i}$

is a functional admitting the group of transformations (2.1) and if $I^{(i)}\left(M_{1}\right.$, ..., $\left.M_{i}\right)$ is the most general symmetric function of $M_{1}, \cdots, M_{i}$ which admits the ith cogrediently extended group of transformations (2.1), then the most general functional of type (2.10) admitting the group of transformations (2.1) can be written as

$$
\begin{aligned}
& \Phi[C]=\phi_{00}^{(0)} \\
& +\sum_{i=1}^{\infty} \frac{1}{i !} \int_{0} \int_{\sigma} \ldots \int_{\sigma} I^{(i)}\left(M_{1}, \cdots, M_{i}\right) \phi_{0}^{(i)}\left(M_{1}, \cdots, M_{i}\right) d \sigma_{1} \cdots d \sigma_{i}
\end{aligned}
$$

$\left(\phi_{00}^{(0)}\right.$ being an arbitrary constant).

Let $\psi^{(i)}\left(M_{1}, \cdots, M_{i}\right)$ be the representative integrand in the most general functional invariant; then by our hypothesis the following equations are satisfied:

$$
\begin{aligned}
& \sum_{j=1}^{i}\left[\xi\left(M_{i}\right) \frac{\partial \phi_{0}^{(i)}}{\partial x_{i}}+\eta\left(M_{j}\right) \frac{\partial \phi_{0}^{(i)}}{\partial y_{j}}+\phi_{0}^{(i)}\left\{\frac{\partial \xi\left(M_{j}\right)}{\partial x_{i}}+\frac{\partial \eta\left(M_{i}\right)}{\partial y_{j}}\right\}\right]=0 \\
& \sum_{i=1}^{i}\left[\xi\left(M_{i}\right) \frac{\partial \psi^{(i)}}{\partial x_{i}}+\eta\left(M_{i}\right) \frac{\partial \psi^{(i)}}{\partial y_{i}}+\psi^{(i)}\left\{\frac{\partial \xi\left(M_{j}\right)}{\partial x_{j}}+\frac{\partial \eta\left(M_{j}\right)}{\partial y_{i}}\right\}\right]=0 .
\end{aligned}
$$

Hence it follows that $\psi^{(i)} / \phi_{0}^{(i)}$ is an invariant function of the $i$ th cogrediently extended group (2.1). Conversely, one can verify readily that if $\psi^{(i)} / \phi_{0}^{(i)}$ is an invariant then the functions $\psi^{(i)}$ will form an invariant functional. The theorem follows therefore readily.

In order to get necessary and sufficient conditions for the invariance of a functional derivative we shall need to study the invariance of functionals $\Phi[C / M]$ depending in particular on a point* $M$ on the curve.

We assume that the variation of a functional $\Phi[C / M]$ has the form

$$
\delta \Phi[C / M]=\frac{\partial \Phi[C / M]}{\partial x} \delta x+\frac{\partial \Phi[C / M]}{\partial y} \delta y+\int_{C} \Phi_{n_{1}^{\prime}}\left[C / M, M_{1}\right] \delta n\left(M_{1}\right) d s_{1}
$$

\footnotetext{
- The point $M$ of course being itself subject to the groups of transformations (2.1).
} 
A necessary and sufficient condition that a functional $\Phi[C / M]$ with a variation of the form (2.20) admit the group of transformations (2.1) is that $\Phi[C / M]$ satisfy the functional equation

$$
\begin{aligned}
& \frac{\partial \Phi[C / M]}{\partial x} \xi(M)+\frac{\partial \Phi[C / M]}{\partial y} \eta(M) \\
+ & \int_{C} \Phi_{n_{1}^{\prime}}\left[C / M, M_{1}\right]\left[\xi\left(M_{1}\right) \cos \left(x_{1}, n_{1}\right)+\eta\left(M_{1}\right) \cos \left(y_{1}, n_{1}\right)\right] d s_{1}=0 .
\end{aligned}
$$

For an explicit solution of our problem we assume $\Phi[C / M]$ to be developable in an expansion

$$
\begin{aligned}
\Phi[C / M]= & \phi^{(0)}(M) \\
& +\sum_{i=1}^{\infty} \frac{1}{i !} \int_{\sigma} \int_{\sigma} \ldots \int_{\sigma} \phi^{(i)}\left(M, M_{1}, \cdots, M_{i}\right) d \sigma_{1} \cdots d \sigma_{i},
\end{aligned}
$$

where $\phi^{(i)}\left(M, M_{1}, \cdots, M_{i}\right)$ is symmetric in the points $M_{1}, M_{2}, \cdots, M_{i}$; possesses continuous first partial derivatives; and

$$
\left|\phi^{(i)}\right|<K \text {. }
$$

On going through a similar analysis to the one used in deriving conditions for the invariance of a functional $\Phi[C]$ of type (2.10) the truth of the following theorem can be verified.

THEOREM 3. A necessary and sufficient condition that a functional $\Phi[C / M]$ possessing a development (2.22) admit the group of transformations defined by (2.1) is that each $\phi^{(i)}\left(M, M_{1}, \cdots, M_{i}\right)$ satisfy the corresponding partial differential equation

$$
\begin{aligned}
& \xi(M) \frac{\partial \phi^{(i)}\left(M, M_{1}, \cdots, M_{i}\right)}{\partial x}+\eta(M) \frac{\partial \phi^{(i)}\left(M, M_{1}, \cdots, M_{i}\right)}{\partial y} \\
& +\sum_{j=1}^{i}\left[\xi\left(M_{i}\right) \frac{\partial \phi^{(i)}\left(M, M_{1}, \cdots, M_{i}\right)}{\partial x_{i}}+\eta\left(M_{i}\right) \frac{\partial \phi^{(i)}\left(M, M_{1}, \cdots, M_{i}\right)}{\partial y_{i}}\right. \\
& \left.+\phi^{(i)}\left(M M_{1}, \cdots, M_{i}\right)\left\{\frac{\partial \xi\left(M_{i}\right)}{\partial x_{j}}+\frac{\partial \eta\left(M_{j}\right)}{\partial y_{j}}\right\}\right]=0(i=0,1,2, \cdots)
\end{aligned}
$$

On comparing the conditions for the invariance of a functional $\Phi[C]$ of type (2.10) and its normal functional derivatives* $\Phi_{n}^{\prime}[C / M]$ we find that the following conditions have to be imposed on the group (2.1):

\footnotetext{
* Such a functional will have the form (2.22) in which all the integrands will be symmetric functions of all their point arguments.
} 


$$
\frac{\partial \xi(M)}{\partial x}+\frac{\partial \eta(M)}{\partial y}=0 .
$$

Hence a necessary and sufficient condition that the normal functional derivative $\Phi_{n}^{\prime}[C / M]$ of a functional $\Phi[C]$ with a development (2.10) which admits the group (2.1) be itself invariant of (2.1) is that the group (2.1) be area preserving.

3. Invariant functionals of closed space curves. The theory given in the preceding paragraph, although directly extensible to functionals of closed surfaces in space, is not amenable to a discussion of functionals of closed curves in space. We shall see, however, that the invariant theory of a certain large class of functionals of closed space curves involves the invariant theory of functionals of closed plane curves as a special case.

Let $F_{x}^{\prime}[C / t], F_{y}^{\prime}[C / t], F_{z}^{\prime}[C / t]$ be the functional derivatives along the $x, y$ and $z$ directions respectively of a functional $F[C]$ of closed space curves $C$. These functional derivatives are taken at a point $M$ on the curve $C$ having $t$ as the corresponding value of the parameter that defines the position of a point on the curve.

We shall deal with functionals whose variation* is given by

$$
\delta F[C]=\int_{C}\left\{F_{x}^{\prime}[C / t] \delta x(t)+F_{y}^{\prime}[C / t] \delta y(t)+F_{z}^{\prime}[C / t] \delta z(t)\right\} d t .
$$

From the well known identity

$$
F_{x}^{\prime} d x+F_{y}^{\prime} d y+F_{z}^{\prime} d z=0
$$

that holds along the curves $C$, Volterra deduced the existence of a functional quantity $V$ (called the flux of the functional $F[C]$ by Paul Lévy) with components $V_{x}, V_{y}, V_{z}$.

In terms of the functional flux $V, \dagger$ the variation (3.1) takes the form

$$
\delta F[C]=\int_{C}\left|\begin{array}{lll}
d x & d y & d z \\
\delta x & \delta y & \delta z \\
V_{x} & V_{y} & V_{z}
\end{array}\right|
$$

Consider a one-parameter group of transformations

$$
\frac{d x}{\xi(M)}=\frac{d y}{\eta(M)}=\frac{d z}{\zeta(M)}=d \tau .
$$

* Cf. Volterra, Acta Mathematica, vol. 12, pp. 237-244. See also Evans, The Cambridge Colloquium, Part I, pp. 8-12, and Hadamard, Leçons sur le Calcul des Variations, pp. 286-287.

f It is easy to see that the functional flux is essentially an alternating covariant tensor. 
A necessary and sufficient condition that a functional $F[C]$ of closed space curves admit a one-parameter group (3.4) is that its functional flux $V$ satisfy the functional equation

$$
\int_{C}\left|\begin{array}{lll}
d x & d y & d z \\
\xi & \eta & \zeta \\
V_{x} & V_{y} & V_{z}
\end{array}\right|=0 .
$$

We note that in the special case in which the curves $C$ are plane, we can take the $x y$ plane as that of the curves $C$. Then if we take one-parameter groups (2.1) in that plane, we are led immediately to the functional equation (2.7).

To get an explicit solution of the functional equation (3.5) we can assume that our functional $F[C]$ is of the form

$$
\phi_{0}+\sum_{i=1}^{\infty} \frac{1}{i !} \int_{\sigma} \int_{\sigma} \cdots \int_{\sigma} \phi_{n_{1} n_{2}} \ldots n_{i}\left(M_{1}, M_{2}, \cdots, M_{i}\right) d \sigma_{1} d \sigma_{2} \cdots d \sigma_{i}
$$

In (3.6), $\phi_{0}$ is a constant;

$$
\begin{aligned}
\phi_{n_{1}}\left(M_{1}\right)= & \psi_{x_{1}}\left(M_{1}\right) \cos \left(x_{1}, n_{1}\right)+\psi_{y_{1}}\left(M_{1}\right) \cos \left(y_{1}, n_{1}\right) \\
& +\psi_{z_{1}}\left(M_{1}\right) \cos \left(z_{1}, n_{1}\right) \\
\phi_{n_{1} n_{1}}\left(M_{1}, M_{2}\right)= & \psi_{x_{1} x_{2}}\left(M_{1}, M_{2}\right) \cos \left(x_{1}, n_{1}\right) \cdot \cos \left(x_{2}, n_{2}\right) \\
& +\psi_{x_{1} y_{2}}\left(M_{1}, M_{2}\right) \cos \left(x_{1}, n_{1}\right) \cdot \cos \left(y_{2}, n_{2}\right) \\
& +\psi_{x_{1} z_{2}}\left(M_{1}, M_{2}\right) \cos \left(x_{1}, n_{1}\right) \cdot \cos \left(z_{2}, n_{2}\right) \\
& +\psi_{y_{1} x_{2}}\left(M_{1}, M_{2}\right) \cos \left(y_{1}, n_{1}\right) \cdot \cos \left(x_{2}, n_{2}\right) \\
& +\psi_{y_{1} y_{2}}\left(M_{1}, M_{2}\right) \cos \left(y_{1}, n_{1}\right) \cdot \cos \left(y_{2}, n_{2}\right) \\
& +\psi_{y_{1} z_{2}}\left(M_{1}, M_{2}\right) \cos \left(y_{1}, n_{1}\right) \cdot \cos \left(z_{2}, n_{2}\right) \\
& +\psi_{z_{1} z_{2}}\left(M_{1}, M_{2}\right) \cos \left(z_{1}, n_{1}\right) \cdot \cos \left(x_{2}, n_{2}\right) \\
& +\psi_{z_{1} y_{3}}\left(M_{1}, M_{2}\right) \cos \left(z_{1}, n_{1}\right) \cdot \cos \left(y_{2}, n_{2}\right) \\
& +\psi_{z_{1} z_{2}}\left(M_{1}, M_{2}\right) \cos \left(z_{1}, n_{1}\right) \cdot \cos \left(z_{2}, n_{2}\right)
\end{aligned}
$$

Furthermore, $\sigma$ is the area of a surface capping the closed curve $C$. The functional (3.6) will depend only on the closed curve $C$ if*

- Cf. Cornelia Fabri, Sopra alcune proprietd generali delle funsioni che dipendono da altre funzioni e da linee, Atti della Reale Accademia delle Scienze di Torino, vol. 25, pp. 671. 


$$
\begin{array}{r}
\frac{\partial \psi_{x_{1}}}{\partial x_{1}}+\frac{\partial \psi_{y_{1}}}{\partial y_{1}}+\frac{\partial \psi_{z_{1}}}{\partial z_{1}}=0, \\
\frac{\partial \psi_{x_{1} x_{2}}}{\partial x_{2}}+\frac{\partial \psi_{x_{1} y_{2}}}{\partial y_{2}}+\frac{\partial \psi_{x_{1} z_{2}}}{\partial z_{2}}=0, \\
\frac{\partial \psi_{y_{1} x_{2}}}{\partial x_{2}}+\frac{\partial \psi_{y_{1} y_{2}}}{\partial y_{2}}+\frac{\partial \psi_{y_{1} z_{2}}}{\partial z_{2}}=0, \\
\frac{\partial \psi_{z_{1} x_{2}}}{\partial x_{2}}+\frac{\partial \psi_{z_{1} y_{2}}}{\partial y_{2}}+\frac{\partial \psi_{z_{1} z_{2}}}{\partial z_{2}}=0, \\
. . . \cdot . \cdot . \cdot .
\end{array}
$$

In (3.6) we have assumed, without any loss of generality, that

$$
\begin{aligned}
& \psi_{x_{1} x_{2}}\left(M_{1}, M_{2}\right)=\psi_{x_{1} x_{2}}\left(M_{2}, M_{1}\right), \\
& \psi_{y_{1} y_{2}}\left(M_{1}, M_{2}\right)=\psi_{y_{1} y_{2}}\left(M_{2}, M_{1}\right), \\
& \psi_{z_{1} z_{2}}\left(M_{1}, M_{2}\right)=\psi_{z_{1} z_{2}}\left(M_{2}, M_{1}\right), \\
& \psi_{x_{1} y_{2}}\left(M_{1}, M_{2}\right)=\psi_{y_{1} x_{2}}\left(M_{2}, M_{1}\right), \\
& \psi_{x_{1} z_{2}}\left(M_{1}, M_{2}\right)=\psi_{z_{1} x_{2}}\left(M_{2}, M_{1}\right), \\
& \psi_{y_{1} z_{2}}\left(M_{1}, M_{2}\right)=\psi_{z_{1} y_{2}}\left(M_{2}, M_{1}\right),
\end{aligned}
$$

We are now in a position to solve the functional equation (3.5) by methods analogous to those given in $\S 2$. The detailed derivation of conditions corresponding to (2.11) entails lengthy calculations. It is here that the tensor analysis enters most appropriately. In fact for functionals* of closed $r$-dimensional manifolds in $n$ dimensions, tensor considerations are a practical necessity.

\section{PART II}

4. Tensors whose components depend on $i$ points. In most textbooks and papers dealing with tensor analysis a tensor is defined as a set of quantities having the following transformation law $\dagger$ under an arbitrary

* Cf. \$11, Part II of this paper.

† From now on a repetition of a Greek letter index in a term will indicate summation from 1 to $n$ unless otherwise stated. 
analytic coördinate transformation $(x)$ to $(\bar{x})$ with non-vanishing jacobian:

$$
\bar{T}_{\lambda \mu}^{\alpha \beta \cdots \sigma}(\bar{x})=T_{v \phi}^{\zeta \eta \cdots \psi}(x) \frac{\partial x^{\nu}}{\partial \bar{x}^{\lambda}} \frac{\partial x^{\phi}}{\partial \bar{x}^{\mu}} \cdots \frac{\partial x^{\psi}}{\partial \bar{x}^{\sigma}} \frac{\partial \bar{x}^{\alpha}}{\partial x^{5}} \frac{\partial \bar{x}^{\beta}}{\partial x^{\eta}} \cdots \frac{\partial \bar{x}^{\epsilon}}{\partial x^{\alpha}} .
$$

Each component of the tensor $T$ in (4.1) is assumed in general to be a point function. More recently Paul Dienes* has studied tensors whose components depend also on a curve in connection with the non-integrable cases of LeviCivita's equations of infinitesimal parallelism. More generally, there are tensors whose components depend also on $r$-dimensional manifolds in $n$ dimensions. Such for example is the case in the flux $\dagger$ of a non-additive functional of closed $r$-dimensional manifolds in $n$ dimensions. Such a flux is essentially a covariant tensor of rank $r+1$ whose components are functionals of the closed $r$-dimensional manifolds depending on a variable point on the manifold.

In the functional invariant theories which I propose to develop now, there occur expressions whose components are functions of $i$ points of the same $n$-dimensional space. Let $\left(x_{k}^{1}, x_{k}^{2}, \cdots, x_{k}^{n}\right)$ be the coördinates of a point $M_{k}$ in a coördinate system $x$ of an $n$-dimensional space. Consider an analytic transformation of coördinates $x$ to $\bar{x}$ with non-vanishing jacobian. A set of quantities

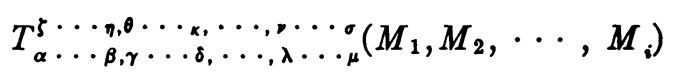

whose law of transformation under the $i$ th cogrediently extended group of analytic transformations is

$$
\begin{aligned}
& \bar{T}_{a \cdots b, c \cdots d, \cdots, l, \ldots m}^{f \cdots, h \ldots k, \ldots, p}\left(\bar{M}_{1}, \bar{M}_{2}, \cdots, \bar{M}_{i}\right)
\end{aligned}
$$

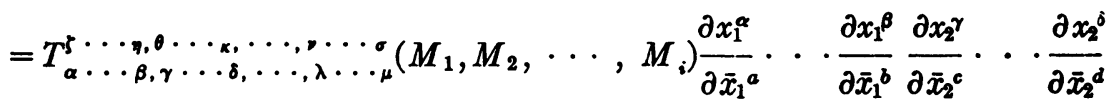

$$
\begin{aligned}
& \cdots \frac{\partial x_{i}^{\lambda}}{\partial \bar{x}^{l}} \cdots \frac{\partial x_{i}^{\mu}}{\partial \bar{x}_{i}^{m}} \frac{\partial \bar{x}_{1}^{b}}{\partial x_{1}^{j}} \cdots \frac{\partial \bar{x}_{1}^{0}}{\partial x_{1}^{\eta}} \cdots \frac{\partial \bar{x}_{i}^{p}}{\partial x_{i}^{p}} \cdots \frac{\partial \bar{x}_{i}^{g}}{\partial x_{i}^{\sigma}},
\end{aligned}
$$
1924.

* Paul Dienes, Sur la structure mathématique du calcul tensoriel, Journal de Mathématiques,

† Volterra's work on functionals of $r$-dimensional manifolds can be considerably simplified and enriched by such a tensor point of view. I hope to take up this study in later papers. 
will be termed a multiple tensor*. We shall speak of the covariant or contravariant rank of such a multiple tensor with respect to each point $M_{k}$. For example, the multiple tensor (4.2) with respect to the point $M_{2}$ is covariant with a rank equal to the number of indices $\gamma$ to $\delta$ and contravariant with a rank equal to the number of indices $\theta$ to $\kappa$.

It is clear from our definition of a multiple tensor that most of the laws of the ordinary tensor algebra can be extended to our case. For example, we shall find useful the following three properties:

(I) If all the components of a multiple tensor vanish identically in a particular coördinate system, they vanish identically in every coördinate system.

(II) If the relation

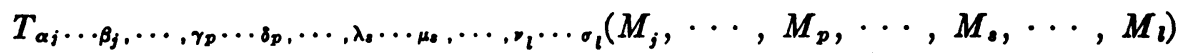

$$
\begin{aligned}
& \text { (4.4) }=T_{\alpha_{j} \cdots \beta_{j}, \cdots, \lambda_{s} \cdots \mu_{s}, \cdots, \gamma_{p} \cdots \delta_{p}, \cdots, p_{l} \cdots \sigma_{l}}\left(M_{j}, \cdots, M_{s}, \cdots, M_{p}, \cdots, M_{l}\right)
\end{aligned}
$$

holds in one coördinate system, it holds in every coördinate system.

(III) If a multiple tensor $T$ is alternating separately in each set of subscripts $\alpha_{i} \cdots \beta_{i}$ to $\nu_{l} \cdots \sigma_{l}$ in one coördinate system, it is in every coördinate system.

Certain multiple tensors will appear frequently in our work, so to escape digression in the coming paragraphs it will be advantageous to discuss them presently. We define the four sets of quantities

$$
\begin{aligned}
& R_{\alpha_{\beta} \beta_{1} \cdots \sigma_{1}, \cdots, \alpha_{k} \beta_{k} \cdots \sigma_{k} \omega} \cdots, \alpha_{i} \cdots \sigma_{i} \\
& R_{\alpha_{1} \cdots \sigma_{1}}^{(k)}, M_{1}, \cdots, \alpha_{i} \cdots \sigma_{i}\left(M_{1}, \cdots, M_{i}\right), \\
& S_{\alpha_{\alpha_{1}} \cdots \rho_{1} \sigma_{1}, \cdots, \alpha_{k} \cdots \rho_{k}^{*}, \cdots, \alpha_{i} \cdots \rho_{i} \sigma_{i}}\left(M_{1}, \cdots, M_{k}, \cdots, M_{i}\right), \\
& S_{\alpha_{1} \cdots \sigma_{1}, \cdots, \alpha_{i} \cdots \sigma_{i}}^{(k)}\left(M_{1}, \cdots, M_{i}\right)
\end{aligned}
$$

in terms of a contravariant vector $X^{\omega}(M)$ and a multiple tensor $T_{\alpha}{ }^{1} \cdots_{\sigma_{1}}, \cdots, \alpha_{i} \ldots \sigma_{i}\left(M_{1}, \cdots, M_{i}\right)$ in the following manner:

$$
\begin{gathered}
R_{\alpha_{1} \beta_{1} \cdots \rho_{1} \sigma_{1}, \cdots, \alpha_{k} \beta_{k} \cdots \rho_{k} \sigma_{k} \omega} \cdots, \alpha_{i} \beta_{i} \cdots \rho_{i \sigma i}\left(M_{1}, \cdots, M_{k}, \cdots, M_{i}\right) \\
=\frac{\partial T_{\alpha_{1} \beta_{1} \cdots \sigma_{1}, \cdots, \alpha_{k} \beta_{k} \cdots \sigma_{k}, \cdots, \alpha_{i} \cdots \sigma_{i}}}{\partial x_{k}^{\omega}}-\frac{\partial T_{\alpha_{1} \cdots \sigma_{1}, \cdots, \omega \beta_{k} \cdots \rho_{k} \sigma_{k}, \cdots, \alpha_{i} \cdots \sigma_{i}}}{\partial x_{k}{ }^{\alpha_{k}}} \\
-\cdots-\frac{\partial T_{\alpha_{1}} \cdots \sigma_{1}, \cdots, \alpha_{k} \beta_{k} \cdots \rho_{k} \omega_{1} \cdots, \alpha_{i} \cdots \sigma_{i}}{\partial x_{k}{ }^{\sigma_{k}}}
\end{gathered}
$$

* It is obvious that such a multiple tensor can be considered as a particular tensor (in the ordinary sense) in an associated manifold of $i n$ dimensions with respect to a certain special group of transformations. Such a point of view, however, is not convenient for our paper. 


$$
\begin{aligned}
& R_{\alpha_{1}}^{(k)} \cdots \sigma_{1}, \cdots, \alpha_{i} \ldots \sigma \cdot\left(M_{1}, \cdots, M_{i}\right) \\
& =X_{(k)}^{\omega} R_{\alpha_{1}}^{\omega} \cdots \sigma_{1}, \cdots, \alpha_{k} \cdots \sigma_{k} \omega, \cdots, \alpha_{i} \cdots \sigma_{i}\left(M_{1}, \cdots, M_{k}, \cdots, M_{i}\right), \\
& S_{\alpha_{1} \cdots \rho_{1} \sigma_{1}, \cdots, \alpha_{k} \cdots \rho_{k}^{*}, \cdots, \alpha_{i} \cdots \rho_{i} \sigma_{i}}\left(M_{1}, \cdots, M_{k}, \cdots, M_{i}\right) \\
& =X_{(k)=\alpha_{1} \cdots \rho_{1} \sigma_{1}, \cdots, \alpha_{k} \cdots \rho_{k} \omega, \cdots, \alpha_{i} \cdots \rho_{i} \sigma_{i}}^{\omega}\left(M_{1}, \cdots, M_{k}, \cdots, M_{i}\right) \text {, } \\
& S_{\alpha_{1} \cdots \sigma_{1}, \cdots, \alpha_{i} \cdots \sigma_{i}}^{(k)}\left(M_{1}, \cdots, M_{i}\right) \\
& =\frac{\partial S_{\alpha_{1} \cdots \sigma_{1}, \cdots, \alpha_{k} \cdots \rho_{k}{ }^{*}, \cdots, \alpha_{i} \cdots \sigma_{i}}}{\partial x_{k}^{\sigma_{k}}}-\frac{\partial S_{\alpha_{1} \cdots \sigma_{1}, \cdots, \sigma_{k} \beta_{k}^{*} \cdots \rho_{k}, \cdots, \alpha_{i} \cdots \sigma_{i}}}{\partial x_{k}^{\alpha_{k}}} \\
& \text { - . . }-\frac{\partial S_{\alpha_{1}} \cdots \sigma_{1}, \cdots, \alpha_{k} \cdots \pi_{k \sigma_{k}}, \cdots, \alpha_{i} \cdots \sigma_{i}}{\partial x_{k} p^{k}} \\
& (k=1,2, \cdots, i) \text {. }
\end{aligned}
$$

In (4.5) we have written $X_{(\boldsymbol{k})}^{\omega}$ as a convenient notation for $X^{\omega}\left(M_{k}\right)$.

If the multiple tensor $T_{\alpha_{1}} \cdots \sigma_{1}, \cdots, \alpha_{i} \cdots \sigma_{i}$ is alternating separately in each of the $i$ sets of subscripts $\alpha_{1} \cdots \sigma_{1}$ to $\alpha_{i} \cdots \sigma_{i}$ and if it satisfies symmetry relations of type (4.4) then the four sets of quantities defined in (4.5) are multiple tensors with corresponding alternating and symmetry relations. This statement follows from a direct calculation of the law of transformation of these four sets of quantities from the known transformation law of the multiple tensor $T$ and the contravariant vector $X$. Furthermore, we note that the symbol $\star$ in $S_{\alpha_{1}} \ldots \sigma_{1}, \cdots, \alpha_{k} \ldots \rho_{k^{*}}, \cdots, \alpha_{i} \ldots \alpha_{i}$ can be considered as on equal footing with the subscripts $\alpha_{k}$ to $\rho_{k}$ with respect to the alternating character of $S$.

5. Functionals involving multiple covariant tensors. Let

$$
x^{i}=f^{i}\left(u_{1}, u_{2}, \cdots, u_{r}\right) \quad(i=1,2, \cdots, n)
$$

be the equations of an $r$-dimensional manifold $S_{r}$ and $D_{r}$ the image of $S_{r}$ on the space of the parameters $u$. As we shall have to deal with functions of several points in space we shall use the notation

$$
x_{k}^{i}=f^{i}\left(u_{k 1}, u_{k 2}, \cdots, u_{k r}\right)
$$

to mean the coördinates of a point $M_{k}$ of the manifold $S_{r}$.

Let

$$
\delta_{l m} x_{p}^{\alpha}=\frac{\partial x_{p}^{\alpha}}{\partial u_{l m}} \delta u_{l m} \quad(p, l=1,2, \ldots, i ; m=1,2, \ldots, r
$$


and in the case of a one-dimensional manifold we shall write

$$
\delta_{l} x_{p}^{\alpha}=\frac{\partial x_{p}^{\alpha}}{\partial u_{l}} \delta u_{l}
$$

Consider the following functional of an $r$-dimensional manifold $S_{r}$ :

$$
\begin{aligned}
& \int_{D_{r}} \int_{D_{r}(i)} \cdots \int_{D_{r}} T_{\alpha_{1} \beta_{1}} \cdots \sigma_{1}, \alpha_{2} \beta_{2} \cdots \sigma_{2}, \cdots, \alpha_{i} \beta_{i} \cdots \sigma_{i}\left(M_{1}, M_{2},\right.
\end{aligned}
$$

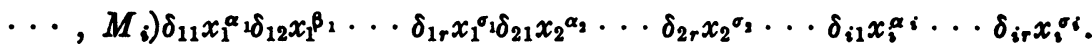

In (5.4) the multiple tensor $T_{\alpha_{1}} \ldots \sigma_{1}, \ldots, \alpha \ldots \sigma_{i}$ is assumed to be alternating in each set of variables $\alpha_{1} \cdots \rho_{1}$ to $\alpha_{i} \cdots \rho_{i}$. Thus the value of (5.4) does not depend on any particular choice of the parameters $u$ but is indeed only dependent on an $r$-dimensional manifold $S_{r}$. We shall assume also, without any loss of generality*, that the multiple covariant tensor $T_{\alpha_{1}} \ldots \sigma_{1}, \ldots, \alpha_{i} \ldots \ldots$, satisfies the symmetry relation of type (4.4). For brevity in writing, we shall sometimes use the notation

$$
\int_{D_{r}}^{(i)} T(i) \delta x
$$

for a functional of type (5.4).

6. Some preliminary material. In $n$ dimensions we consider the oneparameter continuous group of transformations defined by

$$
\frac{d x^{i}}{d \tau}=X^{i}(M) \quad(i=1,2, \cdots, n) .
$$

In (6.1) we assume that the components of the vector $X^{i}$ are analytic functions of their arguments in a certain finite region in which we shall work.

Unless otherwise stated, a total differentiation with respect to the parameter $\tau$ will denote differentiation along the path curves of the group (6.1). Clearly, if the parameters $u$ in (5.4) are taken to be independent of the group parameter $\tau$, we shall have the following system of equations : $\dagger$

* Since the domain of integration $D_{r}$ is the same in each of the $i$ integrations, it follows that the functional can be put in such a form without altering its value.

† Equations (6.2) form a system which is the $i$ th cogrediently extended variational equations of Poincare connected with the system of equations (6.1). 


$$
\begin{aligned}
& \frac{d\left(\delta_{l m} x_{p}^{\alpha}\right)}{d \tau}=\frac{\partial X_{(p)^{\alpha}}^{\alpha}}{\partial x_{p}^{\beta}} \delta_{l m} x_{p}^{\beta}, \\
& \frac{d\left(\delta_{l} x_{p}^{\alpha}\right)}{d \tau}-=\frac{\partial X_{(p)^{\alpha}}}{\partial x_{p}^{\beta}} \delta_{l} x_{p}^{\beta} \quad(p, l=1,2, \cdots, i ; m=1,2, \cdots, r),
\end{aligned}
$$

where we have written $X_{(p)}^{\alpha}$ for $X^{\alpha}\left(M_{p}\right)$.

If $A_{\alpha_{1}} \cdots \sigma_{1}, \cdots, \alpha_{i} \cdots \sigma_{i}\left(M_{1}, \cdots, M_{i}\right)$ is a multiple covariant tensor, we deduce, in virtue of (6.1), the following relations:

$$
\frac{d A_{\alpha_{1} \cdots \sigma_{1}, \cdots, \alpha_{i} \cdots \sigma_{i}}\left(M_{1}, \cdots, M_{i}\right)}{d \tau}=\frac{\partial A_{\alpha_{1} \cdots \sigma_{1}, \cdots, \alpha_{i} \cdots \sigma_{i}}\left(M_{1}, \cdots, M_{i}\right)}{\partial x_{\gamma}^{\beta}} X_{(\gamma)}^{\beta} \text {, }
$$

where $\boldsymbol{\gamma}$ is summed from 1 to $i$.

7. Invariant functionals of one-dimensional manifolds. The functional identities that arise in the invariant theory of functionals of type (5.4) for $r=1$ (one-dimensional manifolds) require a separate treatment from those that arise in the cases $r>1$. In fact the discussion of the case $r=1$ has to be given in two parts. The reason for this will become evident presently.

Let us consider then quadratic functionals (5.4) of one-dimensional manifolds $S_{1}$; i.e., functionals of type

$$
\int_{u_{0}}^{u} \int_{u_{0}}^{u} T_{\alpha, \beta}\left(M_{1}, M_{2}\right) \delta_{1} x_{1}^{\alpha} \delta_{2} x_{2}^{\beta}
$$

In order that the functional (7.1) admit the group of transformations (6.1) it is necessary and sufficient that the total derivative of (7.1) with respect to the parameter $\tau$ be zero for all one-dimensional manifolds. This statement is merely the analytic condition for the following geometrical situation:

Let $\tau=0$ correspond to the position of the one-dimensional manifold $S_{1}$. Consider the transform $S_{1}(\tau)$ of $S_{1}$ by the finite transformations of the group (6.1). In general, a functional (7.1) extended over the image $D_{1}(\tau)$ of $S_{1}(\tau)$ will depend on the group parameter $\tau$. Since the parameters $u$ are chosen independent of $\tau$ we have

$$
\int_{D_{1}(r)}^{(2)}[T(2) \delta x]_{\tau}=\int_{D_{1}}^{(2)}[T(2) \delta x]_{\tau},
$$

where $[T(2) \delta x]_{r}$ is the transform of $T(2) \delta x$ by the finite transformations of the group (6.1). The condition

$$
\int_{D_{1}}^{(2)} T(2) \delta x=\int_{D,}^{(2)}[T(2) \delta x]_{T}
$$


for all values of $\tau$, and that irrespective of the initial one-dimensional manifold $S_{1}$, states the invariance of the functional (7.1) with respect to the one-parameter group defined by (6.1).

Again since the parameter $u$ is chosen independent of $\tau$, the condition for invariance becomes

$$
\int_{u_{0}}^{u} \int_{u_{0}}^{u} \frac{d}{d \tau}\left(T_{\alpha, \beta}\left(M_{1}, M_{2}\right) \delta_{1} x_{1}^{\alpha} \delta_{2} x_{2}^{\beta}\right)=0 .
$$

By (6.1), (6.2), (6.3) and by a rearrangement of the umbral symbols condition (7.2) becomes

$$
\int_{u_{0}}^{u} \int_{u_{0}}^{u}\left(\frac{\partial T_{\alpha, \beta}}{\partial x_{\lambda}^{\gamma}} X_{(\lambda)}^{\gamma}+T_{\gamma, \beta} \frac{\partial X_{(1)}^{\gamma}}{\partial x_{1}^{\alpha}}+T_{\alpha, \gamma} \frac{\partial X_{(2)}^{\gamma}}{\partial x_{2}^{\beta}}\right) \delta_{1} x_{1}^{\alpha} \delta_{2} x_{2}^{\beta}=0
$$

where $\lambda$ is umbral with range 1 to 2 . Since $T_{\alpha, \beta}\left(M_{1}, M_{2}\right)=T_{\beta, \alpha}\left(M_{2}, M_{1}\right)$, we see that the parenthesis in (7.3) is also a multiple tensor $F_{\alpha, \beta}\left(M_{1}, M_{2}\right)$ having the property

$$
F_{\alpha, \beta}\left(M_{1}, M_{2}\right)=F_{\beta, \alpha}\left(M_{2}, M_{1}\right) .
$$

Equation (7.3) is of the form*

$$
\int_{u_{0}}^{u} \int_{u_{0}}^{u} \phi\left(u_{1}, u_{2}\right) \delta u_{1} \delta u_{2}=0
$$

in which $\phi\left(u_{1}, u_{2}\right)$ is a symmetric function of $u_{1}$ and $u_{2}$. By a double use of Leibnitz' formula, once in differentiating partially with respect to $u$ and another time with respect to $u_{0}$, we get

$$
\phi\left(u_{1}, u_{2}\right)=0 .
$$

Hence a necessary and sufficient condition that (7.3) hold is that

$$
\begin{aligned}
\left(\frac{\partial T_{\alpha, \beta}\left(M_{1}, M_{2}\right)}{\partial x_{\lambda} \gamma} X_{(\lambda)}^{\gamma}+T_{\gamma, \beta}\left(M_{1}, M_{2}\right)\right. & \frac{\partial X_{(1)}^{\gamma}}{\partial x_{1}{ }^{\alpha}} \\
& \left.+T_{\alpha, \gamma}\left(M_{1}, M_{2}\right) \frac{\partial X_{(2)}^{\gamma}}{\partial x_{2}{ }^{\beta}}\right) \delta_{1} x_{1} \delta_{2}{ }^{\alpha} x_{2}{ }^{\beta}=0
\end{aligned}
$$

( $\lambda$ umbral with range 1 to 2 )

for all points $M_{1}$ and $M_{2}$ on any arbitrarily given one-dimensional manifold. If we momentarily fix our attention on two arbitrarily given points $M_{1}$ and

* For a given set of functions $x$ the form of a one-dimensional manifold is determined but not its extent. This then means in our problem that for a given set of functions $x$ of the parameter $u$, the interval $\left(u_{0}, u\right)$ in the one-dimensional $u$-space is arbitrary since the extent of the manifold $S$ is arbitrary. 
$M_{2}$, and consider all one-dimensional manifolds passing through $M_{1}$ and $M_{2}$, we see that the infinitesimal displacements $\delta_{1} x_{1}^{\alpha}$ and $\delta_{2} x_{2}^{\beta}$ are arbitrary among themselves and are independent of the $x$ 's, since the direction of the manifold at $M_{1}$ and $M_{2}$ is arbitrary.

From (7.7) then we have the system of equations

$$
\begin{gathered}
\frac{\partial T_{\alpha, \beta}\left(M_{1}, M_{2}\right)}{\partial x_{\lambda}{ }^{\gamma}} X_{\lambda} \gamma+T_{\gamma, \beta}\left(M_{1}, M_{2}\right) \frac{\partial X_{(1)}^{\gamma}}{\partial x_{1}^{\alpha}} \\
+T_{\alpha, \gamma}\left(M_{1}, M_{2}\right) \frac{\partial X_{(2)}^{\gamma}}{\partial x_{2}{ }^{\beta}}=0 \\
(\alpha, \beta=1,2, \cdots, n ; \text { and } \lambda \text { summed from } 1 \text { to } 2) .
\end{gathered}
$$

We shall rewrite (7.8) in a form which will make evident the covariant character of (7.8) by making use of the quantities defined in (4.5). We thus have the conditions

$$
\sum_{\lambda=1}^{2}\left[R_{\alpha, \beta}^{(\lambda)}\left(M_{1}, M_{2}\right)+S_{\alpha, \beta}^{(\lambda)}\left(M_{1}, M_{2}\right)\right]=0 \quad(\alpha, \beta=1,2, \cdots, n) .
$$

Since each of the quantities $R_{\alpha, \beta}^{(1)}, R_{\alpha, \beta}^{(2)}, S_{\alpha, \beta}^{(1)}, S_{\alpha, \beta}^{(2)}$ is a multiple covariant tensor of rank one in $M_{1}$ and rank one in $M_{2}$, it follows that (7.9) is a multiple tensor equation covariant of rank one in $M_{1}$ and of rank one in $M_{2}$.

Thus we have the following theorem:

THEOREM 1. A necessary and sufficient condition that the quadratic functional (7.1) admit the one-parameter group (6.1) is that the components of the multiple tensor $T_{\alpha, \beta}$ satisfy* the tensor equation (7.9).

We turn now to a discussion of the invariance of functionals $F_{i}\left[S_{1}\right]$ of one-dimensional manifolds having the form

$$
\int_{u_{0}}^{u} \int_{u_{0}(i)}^{u} \cdots \int_{u_{0}}^{u} T_{\alpha, \beta}, \cdots, c\left(M_{1}, M_{2}, \cdots, M_{i}\right) \delta_{1} x_{1}^{\alpha} \delta_{2} x_{2}{ }^{\beta} \cdots \delta_{i-1} x_{i-1}^{\theta} \delta_{i} x_{i}
$$

A necessary and sufficient condition that (7.10) admit the group of transformations (6.1) is that

$$
\frac{d}{d \tau} \int_{D_{1}}^{(i)} T(i) \delta x=0
$$

for all one-dimensional manifolds $S$, and hence for all one-dimensional images $D_{1}$.

* The existence and character of the solutions of the system of equations (7.9) will be discussed later. Cf. the discussion for equations (7.17). 
By (6.1), (6.2) and (6.3) and by a rearrangement of the umbral symbols condition (7.11) becomes

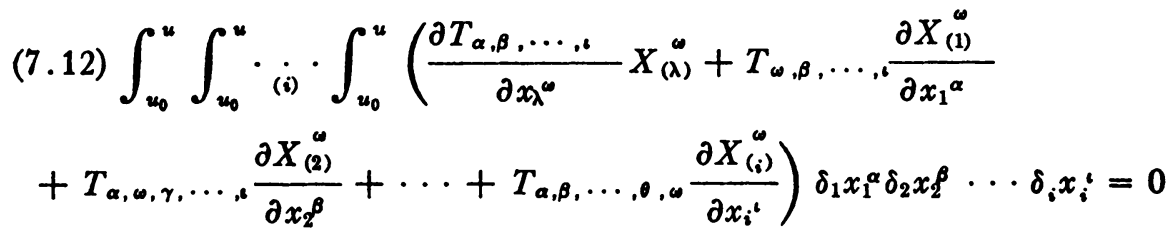

( $\lambda$ umbral with range 1 to $i$ ).

Since the multiple tensor $T_{\alpha, \beta}, \ldots, \downarrow$ has the symmetry properties of type (4.4) it follows that the parenthesis in (7.12) also has the same symmetry relations. Hence (7.12) is an equation of the form

$$
\int_{u_{0}}^{u} \int_{u_{0}}^{u} \cdots \int_{u_{0}}^{u} \phi\left(u_{1}, u_{2}, \cdots, u_{i}\right) \delta u_{1} \delta u_{2} \cdots \delta u_{i}=0 \quad(i>2),
$$

where $\phi$ is a symmetric function of all its $i$ arguments.

A sufficient* condition that (7.13) hold is that

$$
\phi\left(u_{1}, u_{2}, \cdots, u_{i}\right)=0 .
$$

Hence a sufficient condition that (7.12) hold is that

$$
\begin{aligned}
& \left(\frac{\partial T_{\alpha, \beta, \cdots, i}}{\partial x_{\lambda}^{\omega}} X_{(\lambda)}^{\omega}+T_{\omega, \beta, \cdots, i} \frac{\partial X_{(1)}^{\omega}}{\partial x_{1}^{\alpha}}+\cdots+T_{\alpha, \beta, \cdots, 0, \omega} \frac{\partial X_{(i)}^{\omega}}{\partial x_{i}^{\omega}}\right) \\
& \times \delta_{1} x_{1}^{\alpha} \delta_{2} x_{2}^{\beta} \cdots \delta_{i} x_{i}{ }^{c}=0 \quad(\lambda \text { umbral with range } 1 \text { to } i) .
\end{aligned}
$$

Since the direction of the manifold at fixed but arbitrary points $M_{1}$, $M_{2}, \cdots, M_{i}$ is arbitrary we must have

$$
\begin{gathered}
\frac{\partial T_{\alpha, \beta, \cdots, \iota}}{\partial x_{\lambda}^{\omega}} X_{(\lambda)}^{\omega}+T_{\omega, \beta, \cdots, \iota} \frac{\partial X_{(1)}^{\omega}}{\partial x_{1}^{\alpha}}+\cdots+T_{\alpha, \beta, \cdots, 0, \omega} \frac{\partial X_{(i)}^{\omega}}{\partial x_{i}^{c}}=0 \\
(\alpha, \beta, \cdots, \iota=1,2, \cdots, n ; \text { and } \lambda \text { umbral with range } 1 \text { to } i) .
\end{gathered}
$$

We can write condition (7.16) in the form

$$
\text { (7.17) } \begin{array}{r}
\sum_{\lambda=1}^{i}\left[R_{\alpha, \beta, \cdots, 0, \iota}^{(\lambda)}\left(M_{1}, M_{2}, \cdots, M_{i}\right)+S_{\left.\alpha, \beta, \cdots, 0, \iota\left(M_{1}, M_{2}, \cdots, M_{i}\right)\right]=0}^{(\lambda)}(\alpha, \beta, \cdots, \iota=1,2, \cdots, n)\right.
\end{array}
$$

in terms of the multiple tensors defined in (4.5). sort.

* It appears that necessary and sufficient conditions for the condition (7.13) are of a complicated 
Since each $R^{(\lambda)}$ and $S^{(\lambda)}$ in (7.17) is a multiple tensor covariant of rank one in each of the points $M_{1}, M_{2}, \cdots, M_{i}$, it follows that (7.17) is a multiple tensor equation covariant of rank one in each of the points $M_{1}, M_{2}, \cdots, M_{i}$.

It is known* that by a suitable change of coördinate system, say $x$ to $\bar{x}$, the contravariant vector $X$ of the group (6.1) can be made to have components $(0,0, \cdots, 0,1)$ in the new coördinate system $\bar{x}$. In fact, by taking a transformation of coördinates $\dagger$

$$
\bar{x}^{i}=f^{i}\left(x_{1}, x_{2}, \cdots, x^{n}\right) \quad(i=1,2, \cdots, n)
$$

where

$$
f^{i}\left(x^{1}, x^{2}, \cdots, x^{n}\right)=c_{i} \quad(j=1,2, \cdots, n-1)
$$

are $n-1$ functionally independent first integrals of the system of equations (6.1) and

$$
f^{n}\left(x^{1}, x^{2}, \cdots, x^{n}\right)=\tau+c_{n}
$$

is a last integral of (6.1), we see that (6.1) will take the form

$$
\frac{d \bar{x}^{1}}{0}=\frac{d \bar{x}^{2}}{0}=\cdots=\frac{d \bar{x}^{n-1}}{0}=\frac{d \bar{x}^{n}}{1}=d \tau .
$$

Consequently in this new canonical coördinate system $\bar{x}$, the tensor equations (7.17) state that each component of the multiple tensor $\bar{T}_{\alpha, \beta} \ldots \ldots,\left(\bar{M}_{1}, \cdots, \bar{M}_{i}\right)$ must satisfy the partial differential equation

$$
\sum_{\lambda=1}^{i} \frac{\partial f\left(\bar{M}_{1}, \bar{M}_{2}, \cdots, \bar{M}_{i}\right)}{\partial \bar{x}_{\lambda}{ }^{n}}=0 .
$$

The following theorem therefore follows readily.

Theorem 2. A sufficient condition (in the case $i=2$, both a necessary and sufficient condition) that a functional of type (7.10) admit the group of transformations (6.1) is that the components of the multiple tensor $T_{\alpha, \beta, \ldots, .}$ in the canonical coördinate system $\bar{x}$ be functions of the $i$ cogredient sets of $n-1$ first integrals, and of the differences of the cogredient canonical variables $\bar{x}_{\lambda}^{n} ;$ i.e., $\bar{T}$ has the form

$$
\begin{array}{r}
\bar{T}_{\alpha, \beta}, \cdots, l\left(\bar{x}_{1}^{1}, \cdots, \bar{x}_{1}^{n-1}, \bar{x}_{1}^{n}-\bar{x}_{2}^{n}, \bar{x}_{2}^{1}, \cdots, \bar{x}_{2}^{-1}, \bar{x}_{2}^{n}-\bar{x}_{3}^{n}, \cdots,\right. \\
\left.\bar{x}_{i-1}^{n}-\bar{x}_{i}^{n}, \bar{x}_{i}^{1}, \cdots, \bar{x}_{i}^{n-1}\right) .
\end{array}
$$

* This is merely a statement in the language of tensor algebra of the well known fact that all one-parameter groups are similar to a one-parameter group of translations.

† Cf. Goursat, Leçons sur le Problème de Pfaff, \$55. 
8. Invariant functionals of $r$-dimensional manifolds. ${ }^{*}$ We turn now to the invariant theory of functionals having the form (5.4). A necessary and sufficient condition that (5.4) admit the group (6.1) is that along the path curves of (6.1)

$$
\frac{d}{d \tau} \int_{D_{r}}^{(i)} T(i) \delta x=0
$$

for all $\boldsymbol{r}$-dimensional manifolds.

Calculating the condition (8.1) on employing the $i$ th cogrediently extended group of (6.1) and using the relations (5.3), (6.2) and (6.3) we get the condition

$$
\begin{gathered}
\int_{D_{r}} \int_{D r} \cdot \int_{D_{r}}\left(\frac{\partial T_{\alpha_{1} \beta_{1} \cdots \sigma_{1}, \alpha_{2} \cdots \sigma_{2}, \cdots, \alpha_{i} \cdots \sigma_{i}}}{\partial x_{\lambda}^{\omega}} X_{(\lambda)}^{\omega}+T_{\omega \beta_{1} \cdots \sigma_{1}, \cdots, \alpha_{i} \cdots \sigma_{i}} \frac{\partial X_{(1)}^{\omega}}{\partial x_{1}^{\alpha_{1}}}\right. \\
\left.(8.2)+\cdots+T_{\alpha_{1} \cdots \rho_{1} \omega, \cdots, \alpha_{i} \cdots \sigma_{i}}^{\omega} \frac{\partial X_{(1)}^{\omega}}{\partial x_{1} \sigma_{1}}+\cdots+T_{\alpha_{1} \cdots \sigma_{1}, \cdots, \alpha_{i} \cdots \rho_{i}} \frac{\partial X_{(i)}^{\omega}}{\partial x_{i}^{\sigma_{i}}}\right) \\
\quad \times \delta_{11} x_{1}^{\alpha_{1}} \delta_{12} x_{1}{ }^{\beta_{1}} \cdots \delta_{1 r} x_{1} \sigma_{1} \cdots \delta_{i 1} x_{i}^{\alpha_{i}} \cdots \delta_{i r} x_{i}^{\sigma_{i}}=0
\end{gathered}
$$

( $\lambda$ umbral with range 1 to $i$ ).

For a given arbitrary set of functions $x$, equation (8.2) is of the form

$$
\begin{aligned}
\int_{D_{r}} \int_{D_{r(i)}} \cdot \int_{D_{r}} \phi\left(u_{11}, u_{12}, \cdots, u_{1 r} ; u_{21}, \cdots, u_{2 r} ;\right. \\
\left.\cdots ; u_{i 1}, \cdots, u_{i r}\right) \delta u_{11} \cdots \delta u_{i r}=0
\end{aligned}
$$

for arbitrary images $D_{r}$ in the space of the parameters $u$. In (8.3) $\phi$ is symmetric in the $i$ sets of $u$ 's. Hence by an obvious extension of the fundamental lemma of $\$ 2$, we must have $\phi=0$ in the parameters $u$ for any arbitrary given set of functions $x$.

By an evident extension of the corresponding argument in the preceding paragraph it follows that the parenthesis expression in (8.2) must vanish. Employing the multiple tensors defined in $\$ 4$, we can write this condition in the following form:

$$
\begin{aligned}
& \sum_{\lambda=1}^{i}\left[R_{\alpha_{1} \beta_{1} \cdots \sigma_{1}, \alpha_{2} \cdots \sigma_{2}, \cdots, \alpha_{i} \cdots \sigma_{i}}^{(\lambda)}\left(M_{1}, M_{2}, \cdots, M_{i}\right)\right. \\
& (8.4)+S_{\alpha_{1} \beta_{1} \cdots \sigma_{1}, \alpha_{2} \cdots \sigma_{2}, \cdots, \alpha_{i} \cdots \sigma_{i}}^{(\lambda)}\left(M_{1}, M_{2}, \cdots, \begin{array}{r}
\left.\left., M_{i}\right)\right]=0 \\
\left(\alpha_{1}, \cdots, \sigma_{i}=1,2, \cdots, n\right) .
\end{array}\right.
\end{aligned}
$$

* In this paragraph the necessary and sufficient conditions will have reference to the cases $r>1$ and $i=1,2, \cdots$; or to the case $r=1, i=2$. 
The multiple tensor character of equations (8.4) follows from the fact that each $R^{(\lambda)}$ and $S^{(\lambda)}$ is a multiple tensor.

To demonstrate the existence of solutions for the partial differential equations (8.4) and to exhibit the character of these solutions, we consider the tensor equations (8.4) in the coördinate system $\bar{x}$ defined by (7.18). In this special coördinate system $\bar{x}$, conditions (8.4) state that each component of $\bar{T}_{\alpha_{1}} \ldots \sigma_{1}, \ldots, \alpha_{i} \ldots \sigma_{i}\left(\bar{M}_{1}, \ldots, \bar{M}_{i}\right)$ will have to satisfy the same partial differential equation (7.20). We can therefore state the following theorem.

Theorem 3. A necessary and sufficient condition that a functional (5.4) of $r$-dimensional manifolds admit the group of transformations (6.1) is that the components of the multiple tensor $T_{\alpha_{1}} \ldots \sigma_{1}, \ldots, \alpha_{i} \ldots \sigma_{i}$ in the canonical coordinate system $\bar{x}$ be functions of the $i$ cogredient sets of $n-1$ first integrals $\bar{x}_{\lambda}{ }^{\alpha}(\alpha=1, \cdots, n-1)$ and of the differences $\bar{x}_{\lambda-1}^{n}-\bar{x}_{\lambda}{ }^{n}(\lambda=2, \cdots, i)$.

9. Invariant functionals attached to the path curves of a group. Let $\lambda(M)$ be a real analytic function of $x^{1}, x^{2}, \cdots, x^{n}$. Consider the oneparameter group whose infinitesimal transformations* are

$$
\frac{d x^{i}}{d \tau}=\lambda(M) X^{i}(M) \quad(i=1,2, \cdots, n) .
$$

We lay down the following definition.

DEFINITIon. $\dagger A$ functional admitting the infinitesimal transformations (9.1) for all functions $\lambda(M)$ will be called an invariant functional attached to the path curves of the one-parameter group (6.1).

We shall demonstrate the following theorem.

* It is clear that the path curves of the group of transformations (9.1) are the same as those of (6.1) for any function $\lambda(M)$.

$\dagger$ This definition is a generalization of Goursat's definition of integral invariants attached to the trajectories of a system of differential equations (6.1). Cf. Goursat's Leçons sur le Probleme de Pfaff, pp. 236. It is clear from our definition that an invariant functional attached to the path curves of group (6.1) can be considered as a functional which is invariant under the system of differential equations

$$
\frac{d x^{1}}{X^{1}}=\frac{d x^{2}}{X^{2}}=\cdots=\frac{d x^{n}}{X^{n}} .
$$

In other words, then, we are here generalizing certain Cartan invariant theories. For example, equations (9.2) in differential form are the generalizations of characteristic systems of differential forms. Cf. Cartan, Leçons sur les Invariants Intégraux, Chapter IV. 
Theorem 1. Necessary and sufficient conditions that a functional of r-dimensional manifolds (5.4) be an invariant functional attached to the path curves of a one-parameter group of transformations (6.1) are that the following multiple tensor equations hold:

$$
\begin{aligned}
& R_{\alpha_{1} \cdots \sigma_{1}, \cdots, \alpha_{i} \cdots \sigma_{i}}^{(\lambda)}\left(M_{1}, \cdots, M_{i}\right)=0, \\
& S_{\alpha_{1} \cdots \rho_{1} \sigma_{1}, \cdots, \alpha_{k} \cdots \rho_{k}^{*}, \cdots, \alpha_{i} \cdots \rho_{i} \sigma_{i}}\left(M_{1}, \cdots, M_{k}, \cdots, M_{i}\right)=0 \\
& \left(\alpha_{1}, \cdots, \sigma_{i}=1,2, \cdots, n ; k, \lambda=1,2, \cdots, i\right) .
\end{aligned}
$$

Necessary and sufficient conditions that a functional (5.4) be an invariant functional attached to the path curves of (6.1) are that conditions (8.4) hold for all functions $\lambda(M)$ when $\lambda(M) X^{\alpha}(M)$ is substituted for $X^{\alpha}(M)$ in (8.4). On rearranging the terms this condition takes the form

$$
\begin{aligned}
& \sum_{\mu=1}^{i} \lambda\left(M_{\mu}\right)\left[R_{\alpha_{1}}^{(\mu)} \cdots \sigma_{1}, \cdots, \alpha_{i} \cdots \sigma_{i}\left(M_{1}, \cdots, M_{i}\right)\right. \\
& \left.+S_{\alpha_{1}}^{(\mu)} \cdots_{\sigma_{1}}, \ldots, \alpha_{i} \cdots \sigma_{i}\left(M_{1}, \cdots, M_{i}\right)\right] \\
& +S_{\alpha_{1} \beta_{1}} \ldots \rho_{1}{ }^{*}, \cdots, \alpha_{i} \cdots \sigma_{i} \frac{\partial \lambda\left(M_{1}\right)}{\partial x_{1}^{\sigma_{1}}}-S_{\sigma_{1} \beta_{1}} \ldots \rho_{1}^{*}, \cdots, \alpha_{i} \cdots \sigma_{i} \frac{\partial \lambda\left(M_{1}\right)}{\partial x_{1}^{\alpha_{1}}} \\
& -\cdots-S_{\alpha_{1} \beta_{1}} \cdots \pi_{1} \sigma_{1}^{*}, \cdots, \alpha_{i} \cdots \sigma_{i} \frac{\partial \lambda\left(M_{1}\right)}{\partial x_{1}{ }^{1}}+\cdots \\
& +S_{\alpha_{1}} \ldots \sigma_{1}, \ldots, \alpha_{i} \beta_{i} \ldots \rho_{i *}^{*} \frac{\partial \lambda\left(M_{i}\right)}{\partial x_{i}^{\sigma_{i}}}-S_{\alpha_{1}} \ldots \sigma_{1}, \ldots, \sigma_{i} \beta_{i} \cdots \rho_{i}^{*} \frac{\partial \lambda\left(M_{i}\right)}{\partial x_{i}^{\alpha_{i}}} \\
& -\cdots-S_{\alpha_{1}} \ldots \sigma_{1} \cdots, \alpha_{i} \beta_{i} \cdots \pi_{i} \sigma_{i} * \frac{\partial \lambda\left(M_{i}\right)}{\partial x_{i}^{p i}}=0 .
\end{aligned}
$$

Since $S \ldots \ldots \ldots \ldots$ is an alternating multiple tensor separately in the $i$ sets of subscripts and since $\lambda(M)$ is an arbitrary function, conditions (9.3) yield the conditions

$$
\begin{array}{r}
R_{\alpha_{1}}^{(\mu)} \cdots \sigma_{1}, \cdots, \alpha_{i} \cdots \sigma_{i}\left(M_{1}, \cdots, M_{i}\right)+S_{\alpha_{1}}^{(\mu)} \cdots \sigma_{1}, \cdots, \alpha_{i} \cdots \sigma_{i}\left(M_{1}, \cdots, M_{i}\right)=0 \\
\left(\alpha_{1}, \cdots, \sigma_{i}=1,2, \cdots, n ; \mu=1,2, \cdots, i\right)
\end{array}
$$

and conditions (b) of (9.2).

But in virtue of the conditions (b) of (9.2), we have

$$
\begin{array}{r}
S_{\alpha_{1}}^{(\mu)} \cdots \sigma_{1}, \cdots, \alpha_{i} \cdots \sigma_{i}\left(M_{1}, \cdots, M_{i}\right)=0 \\
\left(\alpha_{1}, \cdots, \sigma_{i}=1,2, \cdots, n ; \mu=1,2, \cdots, i\right) .
\end{array}
$$

The theorem follows therefore readily. 
The multiple tensor equations (9.2) in the canonical coördinate system $\bar{x}$ defined by (7.18) take the simple form

$$
\begin{aligned}
& \frac{\partial \bar{T}_{\alpha_{1}} \cdots \sigma_{1}, \cdots, \alpha_{i} \cdots \sigma_{i}\left(\bar{M}_{1}, \cdots, \bar{M}_{i}\right)}{\partial \bar{x}_{\mu}^{n}}=0, \\
& {\left[\bar{T}_{\alpha_{1}} \ldots \sigma_{1}, \cdots, \alpha_{i} \cdots \sigma_{i}\left(\bar{M}_{1}, \cdots, \bar{M}_{i}\right)\right]_{n}=0} \\
& \left(\alpha_{1}, \cdots, \sigma_{i}=1,2, \cdots, n ; \mu=1,2, \cdots, i\right),
\end{aligned}
$$

where $\left[\bar{T}_{\alpha_{1}, \ldots \alpha_{1}, \cdots, \alpha_{i} \ldots \sigma_{i}}\left(\bar{M}_{1}, \cdots, \bar{M}_{i}\right)\right]_{n}$ stands for any one of the components of the multiple tensor $\bar{T}_{\alpha_{1} \cdots \sigma_{1}} \ldots, \alpha_{i} \ldots \sigma_{i}$ in which at least one of the subscripts $\alpha_{1}$ to $\sigma_{i}$ has the value $n$. Hence we have proved the following theorem.

THEOREM 2. A necessary and sufficient condition that a functional (5.4) of r-dimensional manifolds be an invariant functional attached to the path curves of group (6.1) is that the differential form in (5.4) considered in the canonical coördinate system (7.18), be expressible only in terms of the icogredient sets of the $n-1$ first integrals $\bar{x}_{\mu}^{\alpha}(\alpha=1,2, \cdots, n-1)$ and their differentials.

It is interesting to note that the conditions for non-additive functionals are more stringent than for the additive ones in that the first set of conditions (9.4) for the additive case $(i=1)$ state that the functional is an integral invariant of (6.1) while in the case of the non-additive functionals $(i>1)$ a special invariance is implied.

We shall now give a method by means of which we shall be able to derive an invariant functional of $(r-1)$-dimensional manifolds attached to the path curves of (6.1) from a special invariant functional of $r$-dimensional manifolds that is not attached to the path curves of (6.1).

Let

$$
\int_{D_{r}}^{(i)} T(i) \delta x
$$

be an invariant functional of $r$-dimensional manifolds that satisfies the special conditions

$$
\begin{aligned}
& R_{\alpha_{1}}^{(\lambda)} \cdots_{\sigma_{1}, \cdots, \alpha_{i} \ldots \sigma_{i}}\left(M_{1}, \cdots, M_{i}\right)+S_{\alpha_{1} \cdots \sigma_{1}, \cdots, \alpha_{i} \cdots \sigma_{i}}^{(\lambda)}\left(M_{1}, \cdots, M_{i}\right)=0 \\
& \left(\alpha_{1}, \cdots, \sigma_{i}=1,2, \cdots, n ; \lambda=1,2, \cdots, i\right)
\end{aligned}
$$


and is not attached to the path curves of (6.1). We shall show* that the functional of $(r-1)$-dimensional manifolds

$$
\int_{D_{r-1}}^{(i)} T(i) \delta x
$$

is an invariant functional attached to the path curve of (6.1) when the relation between the $T(i)$ in (9.7) and the $T(i)$ in (9.5) is given by

$$
\begin{aligned}
& T_{\alpha_{1}} \cdots \rho_{1}, \cdots, \alpha_{i} \cdots \rho_{i}\left(M, \cdots, M_{i}\right) \\
& \quad=T_{\alpha_{1}} \cdots \rho_{1} \sigma_{1}, \cdots, \alpha_{i} \cdots \rho_{i} \sigma_{i} \\
& \left(M_{1}, \cdots, M_{i}\right) X_{(1)}^{\sigma_{1}} \cdots X_{(i)}^{\sigma_{i}} .
\end{aligned}
$$

This assertion is proved briefly by observing that in the canonical coordinate $\bar{x}$ given by (7.18) the multiple tensor

$$
\bar{T}_{\alpha_{1} \cdots \rho_{1}, \cdots, \alpha_{i} \cdots p_{i}}\left(\bar{M}_{1}, \cdots, \bar{M}_{i}\right)
$$

satisfies conditions of the form (9.4).

In the theory of integral invariants $\dagger$ one finds the following theorem :

$A$ necessary and sufficient condition that an integral invariant be an integral invariant that is attached to the path curves of (6.1) is that its value be identically zero for all r-dimensional manifolds generated by the path curves of (6.1).

Such a theorem, however, does not go through, in general, for the nonadditive functionals that we have been considering. The necessity part of the theorem is obviously satisfied. The sufficiency part, however, does not hold ; for, the identical vanishing of

$$
\begin{gathered}
\int_{P_{r}} \int_{P_{r}} \cdots \int_{P_{r}} T_{\alpha_{1}} \cdots \rho_{1} \sigma_{1}, \cdots, \alpha_{i} \cdots \rho_{i} \sigma_{i}\left(M_{1}, \cdots, M_{i}\right) \frac{\partial x_{1}^{\alpha_{1}}}{\partial u_{11}} \cdots \frac{\partial x_{1}^{\rho_{1}}}{\partial u_{1 q}} X_{(1)}^{\sigma_{1}} \\
\cdots \frac{\partial x_{i}^{\alpha_{i}}}{\partial u_{i 1}} \cdots \frac{\partial x_{i}^{\rho_{i}}}{\partial u_{i q}} X_{(i)}^{\sigma_{i}} \delta u_{11} \cdots \delta u_{1 q} \delta \tau_{1} \cdots \delta u_{i 1} \cdots \delta u_{i q} \delta \tau_{i}
\end{gathered}
$$

for all $r$-dimensional manifolds generated by the path curves of (6.1) (with $P_{r}$ as their images in the $u, \tau$ space) does not necessarily imply conditions (b) of (9.2).

10. Some geometrical considerations. In this paragraph we shall give a geometrical interpretation $\ddagger$ of the process given in the preceding paragraph

- This can be shown by long straightforward calculations; Goursat follows such a process in the special case of integral invariants. We shall give a much shorter proof for the general problem.

† See Goursat, Quelques points de la théorie des invariants intégraux, Journal de Mathématiques, (7), vol. 1 (1915), pp. 246-247.

$\ddagger$ As a special case, of course, we get Goursat's treatment of integral invariants. Cf. his Leçons sur le Probleme de Pfaff, $\$ 62$. 
that yielded an attached from a special* invariant functional. The special invariant functionals have additive properties for certain manifolds; it is precisely this property which makes it possible to get an attached from a special invariant functional.

Suppose then

$$
\int_{D_{r}}^{(i)} T(i) \delta x
$$

is a special invariant functional. Let $W_{r-1}$ be a manifold of $r-1$ dimensions that is not generated by the path curves of the group (6.1). Each point $a_{0}$ of $W_{r-1}$ is taken contemporaneously, say for $\tau=0$ (for the convenience of exposition we have the kinematical picture in mind). Each point $a_{0}$ of $W_{r-1}$ is taken along the path curves of (6.1) and moved up to a point $a_{r^{\prime}}$, while $\tau$ varies from 0 to $\tau^{\prime}$. The points $a_{r^{\prime}}$ constitute a manifold $W_{r-1}^{\prime}$. Let $W_{r}$ be the manifold generated by the path curves which issue from the points of $W_{r-1}$ and terminate at the corresponding points of $W_{r-1}^{\prime}$ Let $P_{r-1}$ be the manifold which is generated by the path curves issuing from the points of $W_{r-2}$, the boundary of $W_{r-1}$, and terminating at the corresponding points of $W_{r-2}^{\prime}$, the boundary of $W_{r-1}^{\prime}$. Suppose then the coördinates of the points $a_{0}$ of $W_{r-1}$ expressed as functions of the independent variables $u_{1}, u_{2}, \cdots, u_{r-1}$ so that to each point of $W_{r-1}$ corresponds a point in a domain $D_{r-1}(0)$ of the $u$ space and conversely. Hence in $W_{r}$ each point will be a function of $u_{1}, u_{2}, \cdots, u_{r-1}, \tau$, and at each point of $W_{r}$ we shall have

$$
\frac{\partial x^{\alpha}}{\partial \tau}=X^{\alpha}(M) \quad(\alpha=1,2, \cdots, n) .
$$

Our functional (10.1) therefore can be written as

$$
\begin{aligned}
& \int_{0}^{r^{\prime}} \delta \tau_{1} \int_{0}^{\tau^{\prime}} \delta \tau_{2} \cdots \int_{0}^{\tau^{\prime}} \delta \tau_{i} \int_{D r-1\left(\tau_{1}\right)} \int_{D r-1\left(\tau_{2}\right)} \\
& \quad \cdots \int_{D r-1\left(\tau_{i}\right)} T_{\alpha_{1}} \ldots \rho_{1}, \cdots, \alpha_{i} \cdots \rho_{i}\left(M_{1}\right. \\
& \left.\quad \cdots, M_{i}\right) \delta_{11} x_{1}^{\alpha_{1}} \delta_{12} x_{1}^{\beta_{1}} \cdots \delta_{1 r-1} x_{1}^{\rho_{1}} \cdots \delta_{i 1} x_{i}^{\alpha i} \cdots \delta_{i r-1} x_{i}^{\rho_{i}}
\end{aligned}
$$

where the multiple tensor $T_{\alpha_{1} \ldots \rho_{1}} \ldots \ldots \alpha_{i} \ldots \rho_{i}$ is defined as in (9.8).

* By a special invariant functional of $r$-dimensional manifolds (5.4) we shall understand a functional that satisfies the special conditions for invariance (9.6). 
In the above expression we have written $D_{r-1}(\tau)$ to show the dependence of the domain $D_{r-1}$ on $\tau\left(D_{r-1}(0)\right.$ corresponding to $\left.W_{r-1}\right)$. The $i$ th derivative of (10.3) with respect to $\tau^{\prime}$ at $\tau^{\prime}=0$ yields

$$
i ! \int_{D r-1(0)}^{(i)} T(i) \delta x .
$$

Let $\tau^{*}$ be a continuous function of position of the points of $a_{0}$ of $W_{r-1}$. On the path curve issuing from the general point $a_{0}$ of $W_{r-1}$ consider points $a_{\tau^{*}}$ and $a_{\tau^{\prime}+\tau^{*}}$ corresponding to the position of the point $a_{0}$ at times $\tau=\tau^{*}$ and $\tau=\tau^{*}+\tau^{\prime}$ respectively. Thus corresponding to $\tau=0, \tau=\tau^{\prime}, \tau=\tau^{*}, \tau=\tau^{*}+\tau^{\prime}$ there are four manifolds $W_{r-1}, W_{r-1}^{\prime}, W_{r-1}^{*}, W_{r-1}^{* \prime}$ whose representative points are $a_{0}, a_{\tau^{\prime}}, a_{\tau^{*}}, a_{\tau^{*}+\tau^{\prime}}$ respectively. $W_{r-1}^{\prime}$ is the transform of $W_{r-1}$ and $W_{r-1}^{* \prime}$ of $W_{r-1}^{*}$. Thus $W_{r-1}^{*}$ and $W_{r-1}^{* \prime}$ are sections of the tube of path curves whose points are not contemporaneous while those of $W_{r-1}$ and $W_{r-1}^{\prime}$ are contemporaneous. Evidently then by varying the function $\tau$ we get various noncontemporaneous $(r-1)$-dimensional sections of the tube of path curves. The manifold $W_{r}^{*}$ will be defined as that bounded by $W_{r-1}^{*}$ and $W_{r-1}^{* \prime}$; and $P_{r-1}^{*}$, as the manifold which is constituted of the path curves issuing from the points of $W_{r-2}^{*}$, the boundary of $W_{r-1}^{*}$, and terminating at the corresponding points of $W_{r-2}^{* \prime}$, the boundary of $W_{r-1}^{* \prime}$. Furthermore, let $w_{r}$ be the manifold enclosed by $W_{r-1}, W_{r-1}^{*}$ and the path curves issuing from $W_{r-2}$ to $W_{r-2}^{*}$; and $w_{r}^{*}$, the manifold bounded by $W_{r-1}^{\prime}, W_{r-1}^{* \prime}$ and the path curves issuing from $W_{r-2}^{\prime}$ to $W_{r-2}^{* \prime}$. Clearly, the manifold $w_{r}^{*}$ is the transform of $w_{r}$ by the group (6.1). We have

$$
W_{r}^{*}=W_{r}+w_{r}^{*}-w_{r} .
$$

Employing this relation the following steps in the reasoning are clear in the case of a special invariant functional of $\boldsymbol{r}$-dimensional manifolds :

$$
\begin{aligned}
& \int_{W_{r^{*}}} \int_{W_{r^{*}}} \cdots \int_{W_{r^{*}}}=\int_{W_{r^{*}}} \int_{W_{r^{*}}} \cdots \int_{W_{r^{*}}} \int_{W_{r}+w_{r^{*}-w_{r}}} \\
= & \int_{W_{r^{*}}} \cdots \int_{W_{r^{*}}} \int_{W_{r}}+\int_{W_{r^{*}}} \cdots \int_{W_{r^{*}}} \int_{w_{r^{*}}}-\int_{W_{r^{*}}} \cdots \int_{W_{r^{*}}} \int_{W_{r}} \\
= & \int_{W_{r^{*}}} \cdots \int_{W_{r^{*}}} \int_{W_{r}} .
\end{aligned}
$$

Repeating this procedure $i-1$ more times we shall get finally

$$
\int_{W_{r^{*}}(i)} \cdot \int_{W_{r^{*}}}=\int_{W_{r}} \underset{(i)}{ } \int_{W_{r}} \text {. }
$$


Hence the $i$ th derivative with respect to $\tau^{\prime}$ for $\tau^{\prime}=0$ of both sides of (10.6) must be equal. This then means, by (10.4), that

$$
\int_{D r-1\left(r^{*}\right)}^{(i)} T(i) \delta x=\int_{D r-1(0)}^{(i)} T(i) \delta x
$$

where $D_{r-1}(0)$ corresponds to a given arbitrary contemporaneous section of the tube of path curves while $D_{r-1}\left(\tau^{*}\right)$ corresponds, in general, to a noncontemporaneous section. But the function $\tau^{*}$ is arbitrary, and therefore, in virtue of (10.7), the functional of $(r-1)$-dimensional manifolds

$$
\int_{D r-1}^{(i)} T(i) \delta x
$$

has a constant value for all $(r-1)$-dimensional sections (that are not generated by the path curves), whether contemporaneous or not, of any given but arbitrary tube of path curves. But the multiple tensor $T$ in (10.8) is precisely given by (9.8). Hence we have given a geometrical interpretation of the method, given in $\S 9$, that enabled us to step down $\dagger$ from a special invariant functional to an invariant functional that is attached to the path curves of (6.1).

11. Invariant functionals of closed manifolds. The invariant theory of functionals of closed manifolds can easily be referred to the invariant theory of functionals of open manifolds. A functional of closed $(r-1)$-dimensional manifolds

$$
\int_{D r-1}^{(i)} T(i) \delta x
$$

can be thrown into the form of a functional of open $r$-dimensional manifolds

$$
\int_{D r}^{(i)} T(i) \delta x
$$

by applying Stokes's theorem $i$ times. Thus by applying our previous theory to the functional (11.2) we can get necessary and sufficient conditions for the invariance of (11.1).

We can, however, start with a functional (11.2) of open $r$-dimensional manifolds and then impose the condition that it depend only on the bounding $(r-1)$-dimensional manifolds. Since $R_{\alpha_{1}} \ldots \sigma_{1} \omega, \alpha_{2} \cdots \sigma_{2}, \cdots, \alpha_{i} \ldots \sigma_{i}\left(M_{1}, M_{2}, \cdots\right.$,

$\dagger$ This procedure can be considerably generalized when the totality of path curves of the group (6.1) are left invariant by known infinitesimal transformations. I propose to take up these considerations elsewhere. 
$M_{i}$ ) has the skew-symmetric properties discussed in $\$ 4$, it follows that the conditions

$$
\begin{array}{r}
R_{\alpha_{1}} \cdots \sigma_{\sigma_{1} \omega, \alpha_{2}} \cdots \sigma_{2}, \cdots, \alpha_{i} \cdots \sigma_{i}\left(M_{1}, M_{2}, \cdots, M_{i}\right)=0 \\
\left(\alpha_{1}, \cdots, \sigma_{1}, \omega, \alpha_{2}, \cdots, \sigma_{i}=1,2, \cdots, n\right)
\end{array}
$$

are sufficient* to insure the dependence of (11.2) only on the bounding $(r-1)$-dimensional manifold.

In order that a functional (11.2) of closed $(r-1)$-dimensional manifolds which satisfies conditions (11.3) be an invariant functional attached to the path curves of (6.1), it is necessary and sufficient that the linear homogeneous algebraic equations

$$
\begin{array}{r}
T_{\omega \beta_{1}} \cdots \sigma_{1}, \alpha_{2} \beta_{2} \cdots \sigma_{2}, \cdots, \alpha_{i} \cdots \sigma_{i}\left(M_{1}, M_{2}, \cdots, M_{i}\right) X_{(1)}^{\omega}=0 \\
\left(\beta_{1}, \cdots, \sigma_{i}=1,2, \cdots, n\right)
\end{array}
$$

be satisfied by the multiple tensor $T_{\alpha_{1} \cdots \sigma_{1}, \cdots, \alpha_{i} \cdots \sigma_{i}}\left(M_{1}, \cdots, M_{i}\right)$.

12. Complete invariant functionals of manifolds. Up to this point we have been engaged in generalizations of the Poincare integral invariant. It is possible however to generalize Cartan's $\dagger$ complete integral invariants. Goursatł has shown how Cartan's complete integral invariants are bound up with certain Poincare integral invariants that are attached to the path curves of differential equations. As a matter of fact Cartan's complete integral invariants are special Poincaré integral invariants attached to the path curves of the one-parameter group

$$
\frac{d x^{1}}{X^{1}(M)}=\frac{d x^{2}}{X^{2}(M)}=\cdots=\frac{d x^{n}}{X^{n}(M)}=\frac{d \tau}{1}=d \psi
$$

in the space time continuum $\left(x^{1}, x^{2}, \cdots, x^{n}, \tau\right)$. The parameter of the group (12.1) is $\psi$ and $M$ is a point of the $n$-dimensional space $\left(x^{1}, x^{2}, \cdots, x^{n}\right)$.

Cartan has given a method by means of which one can write down a complete integral invariant corresponding to every Poincaré integral invariant. For example, if

$$
\int T_{\alpha} \delta x^{\alpha}
$$

\footnotetext{
* For functionals of closed curves in three dimensions, cf. Cornelia Fabri, Sopra alcune proprietd generali delle funzioni che dipendono da altre funzioni e da linee, Atti della Reale Accademia delle Scienze di Torino, vol. 25, pp. 671.

† Cf. Cartan, Leçons sur les Invariants Intégraux.

$\ddagger$ Comptes Rendus, vol. 174 (1922), pp. 1089-1091.
} 
is an absolute integral invariant of

$$
\frac{d x^{i}}{d \tau}=X^{i}(M) \quad(i=1,2, \cdots, n)
$$

then

$$
\int T_{\alpha} \delta x^{\alpha}-T_{\alpha} X^{\alpha} \delta \tau
$$

is an absolute complete integral invariant.

It is always possible to pass from a Poincaré integral invariant to a corresponding Cartan complete integral invariant. Such, however, is not, in general, the state of affairs when one considers non-additive functional invariants.

In summations it is convenient to replace the variable $\tau$ by $x^{n+1}$. We can rewrite (12.1) therefore in the form

$$
\frac{d x^{\alpha^{\prime}}}{d \psi}=X^{\alpha^{\prime}} \quad\left(\alpha^{\prime}=1,2, \cdots, n+1\right) .
$$

In (12.3) the contravariant vector $X^{\alpha^{\prime}}$ has components $X^{1}\left(x^{1}, x^{2}, \cdots, x^{n}\right)$, $X^{2}\left(x^{1}, \cdots, x^{n}\right), \cdots, X^{n}\left(x^{1}, \cdots, x^{n}\right), 1$. We shall use primed letters for indices when the range of the indices is $1,2, \cdots, n+1$; and unprimed, when the range is 1 to $n$.

Consider the functional of $r$-dimensional manifolds in the space continuum $\left(x^{1}, x^{2}, \cdots, x^{n}\right)$

$$
\int_{D_{r}}^{(i)} T(i) \delta x
$$

Associated with the functional (12.4) we shall define a particular functional of $r$-dimensional manifolds in the space-time continuum $\left(x^{1}, \cdots, x^{n}\right.$, $\left.x^{n+1}\right)$ in the following manner.

Let

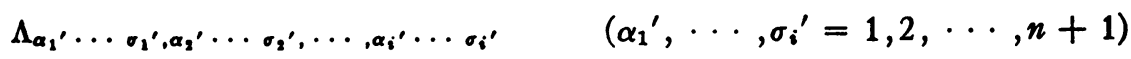

be a multiple tensor whose components are given as follows :

$$
\begin{aligned}
& \Lambda_{\alpha_{1}} \ldots \sigma_{1}, \alpha_{1} \ldots \sigma_{2}, \cdots, \alpha_{i} \cdots \sigma_{i}=T_{\alpha_{1}} \cdots \sigma_{1}, \alpha_{2} \cdots \sigma_{2}, \cdots, \alpha_{i} \cdots \sigma_{i}, \\
& \Lambda_{\alpha_{1}} \ldots \sigma_{1} \ldots,,^{*}(n+1) j_{1}^{*}, \ldots,,^{*}(n+1) j_{2}^{*}, \ldots,{ }^{*}(n+1) j_{k}{ }^{*}, \ldots, \alpha_{i} \cdots \sigma_{i} \\
& \text { (12.5) }=(-1)^{k} T_{\alpha_{1}} \cdots \sigma_{1},{ }^{*} \omega_{j_{1}}{ }^{*}, \ldots, \omega_{j_{2}}{ }^{*}, \ldots,{ }^{*} \omega_{j_{k}}{ }^{*}, \alpha_{i} \cdots \sigma_{i} X_{\left(j_{1}\right)}^{\omega_{j_{1}}} X_{\left(j_{2}\right)}^{\omega_{j_{2}}} \cdots X_{\left(j_{k}\right)}^{\omega_{j_{j}},} \\
& \Lambda_{\alpha_{1}{ }^{\prime} \cdots \sigma_{1^{\prime}}, \ldots, \alpha_{i}{ }^{\prime} \cdots \sigma_{i^{\prime}}}=0 \text {, }
\end{aligned}
$$


whenever two or more subscripts, in any one of the $i$ sets $\alpha_{1}^{\prime} \cdots \sigma_{1}^{\prime}, \cdots, \alpha_{i}^{\prime}$ $\cdots \sigma_{i}^{\prime}$, take on the value $n+1$.

The stars $\star$ indicate undetermined indices which may have any value from 1 to $n$.

The functional of $r$-dimensional manifolds in the space-time continuum $\left(x^{1}, x^{2}, \cdots, x^{n+1}\right)$

$$
\int_{D_{r}} \int_{D_{r}} \cdots \int_{D_{r}} \Lambda_{\alpha_{1}{ }^{\prime} \cdots \sigma_{1^{\prime}}, \cdots, \alpha_{i^{\prime}} \cdots \sigma_{i}{ }^{\prime} \delta_{11} x_{1}^{\alpha_{1}{ }^{\prime}} \cdots \delta_{i r} x_{i}{ }^{\sigma^{\prime}}}
$$

is precisely the functional formed by putting

$$
\delta_{l m} x_{p}^{q}-X_{p}^{q} \delta_{l m} x_{p}^{n+1} \text { for } \delta_{l m} x_{p}^{q} \quad \text { in (12.4) }
$$

By calculation one finds that necessary and sufficient conditions that (12.6) be a functional attached to the path curves of the one-parameter group (12.3) are that the equations (9.6) hold. This is verified by employing conditions (9.2) corresponding to the multiple tensor $\Lambda$ and noting that conditions (b) of (9.2) for $\Lambda_{\alpha_{1}{ }^{\prime}} \cdots \sigma_{1_{1}}, \cdots, \alpha_{i^{\prime}} \cdots \sigma_{i^{\prime}}$ are automatically satisfied. The only conditions on $T_{\alpha_{1}} \cdots_{\sigma_{1}}, \ldots, \alpha_{i} \cdots \sigma_{i}$ are therefore conditions (a) of

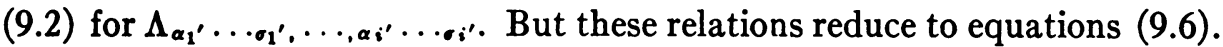

To make the argument clear I shall give the details explicitly in the case of a quadratic functional of two-dimensional manifolds

$$
\int_{D_{2}} \int_{D_{2}} T_{\alpha_{1} \beta_{1}, \alpha_{2} \beta_{2}}\left(M_{1}, M_{2}\right) \frac{\partial x_{1}^{\alpha_{1}}}{\partial u_{11}} \frac{\partial x_{1}^{\beta_{1}}}{\partial u_{12}} \frac{\partial x_{2}^{\alpha}}{\partial u_{21}} \frac{\partial x_{2}^{\beta_{2}}}{\partial u_{22}} d u_{11} d u_{12} d u_{21} d u_{22} .
$$

We consider the functional

$$
\begin{aligned}
& \int_{\mathcal{D}_{2}} \int_{\mathcal{D}_{2}} T_{\alpha_{1} \beta_{1}, \alpha_{2} \beta_{2}}\left(M_{1}, M_{2}\right)\left(\frac{\partial x_{1}^{\alpha_{1}}}{\partial u_{11}}-X_{(1)}^{\alpha_{1}} \frac{\partial x_{1}^{n+1}}{\partial u_{11}}\right)\left(\frac{\partial x_{1}^{\beta_{1}}}{\partial u_{12}}\right. \\
& \left.\left.-X_{(1)}^{\beta_{1} \partial x_{1}^{n+1}} \frac{\partial x_{2}^{\alpha_{2}}}{\partial u_{12}}\right)\left(\frac{\left.X_{(2)}^{\alpha_{2}} \frac{\partial x_{2}{ }^{n+1}}{\partial u_{21}}\right)\left(\frac{\partial x_{2}^{\beta_{2}}}{\partial u_{22}}\right.}{\partial u_{21}}\right) \frac{X_{(2)}^{\beta_{2} \partial x_{2}^{n+1}}}{\partial u_{22}}\right) d u_{11} d u_{12} d u_{21} d u_{22} .
\end{aligned}
$$

We can write (12.8) in the form

$$
\int_{D_{2}} \int_{D_{2}} \Lambda_{\alpha_{1}{ }^{\prime} \beta^{\prime}, \alpha_{2}^{\prime} \beta_{2}^{\prime}} \frac{\partial x_{1}^{\alpha_{1}^{\prime}}}{\partial u_{11}} \frac{\partial x_{1}^{\beta_{1}^{\prime}}}{\partial u_{12}} \frac{\partial x_{2}^{\alpha}{ }^{\prime}}{\partial u_{21}} \frac{\partial x_{2}^{\beta_{2}^{\prime}}}{\partial u_{22}} d u_{11} d u_{12} d u_{21} d u_{22}
$$


where

$$
\begin{array}{ll}
\Lambda_{\alpha_{1} \beta_{1}, \alpha_{2} \beta_{2}}=T_{\alpha_{1} \beta_{1}, \alpha_{2} \beta_{2}}, & \Lambda_{n+1 \beta_{1}, n+1 \beta_{2}}=T_{\alpha_{1} \beta_{1}, \alpha_{2} \beta_{2}} X_{(1)}^{\alpha_{1}} X_{(2)}^{\alpha_{2}}, \\
\Lambda_{n+1 \beta_{1}, \alpha_{2} \beta_{2}}=-T_{\alpha_{1} \beta_{1}, \alpha_{2} \beta_{2}} X_{(1)}^{\alpha_{1}}, & \Lambda_{n+1 \beta_{1}, \alpha_{2} n+1}=T_{\alpha_{1} \beta_{1}, \alpha_{2} \beta_{2}} X_{(1)}^{\alpha_{1}} X_{(2)}^{\beta_{2}}, \\
\Lambda_{\alpha_{1} n+1, \alpha_{2} \beta_{2}}=-T_{\alpha_{1} \beta_{1}, \alpha_{2} \beta_{2}} X_{(1)}^{\beta_{1}}, & \Lambda_{\alpha_{1} n+1, n+1 \beta_{2}}=T_{\alpha_{1} \beta_{1}, \alpha_{2} \beta_{2}} X_{(1)}^{\beta_{1}} X_{(2)}^{\alpha_{2}}, \\
\Lambda_{\alpha_{1} \beta_{1}, n+1 \beta_{2}}=-T_{\alpha_{1} \beta_{1}} X_{(2)}^{\alpha_{2}}, & \Lambda_{\alpha_{1} n+1, \alpha_{2} n+1}=T_{\alpha_{1} \beta_{1}, \alpha_{2} \beta_{2}} X_{(1)}^{\beta_{1}} X_{(2)}^{\beta_{2}}, \\
\Lambda_{\alpha_{1} \beta_{1}, \alpha_{2} n+1}=-T_{\alpha_{1} \beta_{1}, \alpha_{2} \beta_{2}} X_{(2)}^{\beta_{2}}, & \Lambda_{n+1 n+1, \alpha_{2}^{\prime} \beta_{2}^{\prime}}=0, \\
\Lambda_{\alpha_{1}^{\prime} \beta_{1}, n+1 n+1}=0 .
\end{array}
$$

In order that (12.9) be an invariant functional attached to the path curves of the one-parameter group (12.3) it is necessary and sufficient that

$$
\begin{aligned}
& \text { (a) } X_{(1)}^{\omega^{\prime}}\left(\frac{\partial \Lambda_{\alpha_{1}^{\prime} \beta_{1}^{\prime}, \alpha_{2}^{\prime} \beta_{2}^{\prime}}}{\partial x_{1}^{\omega^{\prime}}}-\frac{\partial \Lambda_{\omega^{\prime} \beta_{1}^{\prime}, \alpha_{2}^{\prime} \beta_{2}^{\prime}}}{\partial x_{1}^{\alpha_{1}}{ }^{\prime}}-\frac{\partial \Lambda_{\alpha_{1}^{\prime} \omega^{\prime}, \alpha_{2} \beta_{2}^{\prime}}}{\partial x_{1} \beta_{1}^{\prime}}\right)=0, \\
& \text { (b) } X_{(1)}^{\omega^{\prime}} \Lambda_{\omega^{\prime} \beta_{1}^{\prime}, \alpha_{2}^{\prime} \beta_{2}^{\prime}}=0 .
\end{aligned}
$$

We have made use of the fact that

$$
\Lambda_{\alpha_{1}^{\prime} \beta_{1}^{\prime}, \alpha_{2}^{\prime} \beta_{2}^{\prime}}\left(M_{1}, M_{2}\right)=\Lambda_{\alpha_{2}^{\prime} \beta_{2}^{\prime}, \alpha_{1}^{\prime} \beta_{1}^{\prime}}\left(M_{2}, M_{1}\right)
$$

and that $\Lambda_{\alpha_{1}{ }^{\prime} \beta_{1^{\prime}}, \alpha_{2}{ }^{\prime} \beta_{2^{\prime}}}$ is alternating separately in each of the two sets $\alpha_{1}^{\prime}, \beta_{1}^{\prime}$ and $\alpha_{2}^{\prime}, \beta_{2}^{\prime}$.

The conditions (b) of (12.11) are automatically satisfied; for, from (12.10) we have

$$
X_{(1)}^{\omega^{\prime \prime}} \Lambda_{\omega^{\prime} \beta_{1}, \alpha_{2} \beta_{2}}=X_{(1)}^{\omega} \Lambda_{\omega \beta_{1}, \alpha_{2} \beta_{2}}+\Lambda_{n+1 \beta_{1}, \alpha_{2} \beta_{2}} \equiv 0,
$$

Since

$$
\frac{\partial \Lambda_{\alpha_{1}^{\prime} \beta_{1}^{\prime}, \alpha_{2}^{\prime} \beta_{2}^{\prime}}}{\partial x_{1}^{n+1}}=0,
$$

we can write (a) of (12.11) as

$$
\begin{gathered}
X_{(1)}^{\omega}\left(\frac{\partial \Lambda_{\alpha_{1}^{\prime} \beta_{1}^{\prime}, \alpha_{2}^{\prime} \beta_{2}^{\prime}}}{\partial x_{1}^{\prime}}-\frac{\partial \Lambda_{\omega \beta_{1}^{\prime}, \alpha_{2}^{\prime} \beta_{2}^{\prime}}}{\partial x_{1} \alpha_{1}^{\prime}}-\frac{\partial \Lambda_{\alpha_{1}^{\prime} \omega, \alpha_{2} \beta_{2}^{\prime}}}{\partial x_{1} \beta_{1}^{\prime}}\right) \\
+\frac{\partial \Lambda_{\beta_{1}^{\prime} n+1, \alpha_{2}^{\prime} \beta_{2}^{\prime}}}{\partial x_{1}^{\alpha_{2}^{\prime}}}-\frac{\partial \Lambda_{\alpha_{1}^{\prime} n+1, \alpha_{2}^{\prime} \beta_{2}^{\prime}}}{\partial x_{1} \beta_{1}^{\prime}}=0 .
\end{gathered}
$$


If $\alpha_{1}^{\prime}, \beta_{1}^{\prime}, \alpha_{2}^{\prime}, \beta_{2}^{\prime} \neq n+1$ in (12.14) we have

$$
\begin{gathered}
X_{(1)}^{\omega}\left(\frac{\partial T_{\alpha_{1} \beta_{1}, \alpha_{2} \beta_{2}}}{\partial x_{1}^{\omega}}-\frac{\partial T_{\omega \beta_{1}, \alpha_{2} \beta_{2}}}{\partial x_{1}^{\alpha_{1}}}-\frac{\partial T_{\alpha_{1} \omega, \alpha_{2} \beta_{2}}}{\partial x_{1} \beta_{1}}\right)+\frac{\partial\left(T_{\alpha_{1} \omega, \alpha_{2} \beta_{2}} X_{(1)}^{\omega}\right)}{\partial x_{1} \beta_{1}} \\
-\frac{\partial\left(T_{\beta_{1} \omega, \alpha_{2} \beta_{2}} X_{(1)}^{\omega}\right)}{\partial x_{1}^{\alpha_{1}}}=0 .
\end{gathered}
$$

The other conditions arising from (12.14) are consequences of (12.15), (12.13) and of (12.10). For example, if $\alpha_{1}^{\prime}=n+1 ; \alpha_{2}^{\prime}, \beta_{1}^{\prime}, \beta_{2}^{\prime} \neq n+1$, we have

$$
X_{(1)}^{\omega}\left(\frac{\partial\left(T_{\alpha_{1} \omega, \alpha_{2} \beta_{2}} X_{(1)}^{\alpha_{1}}\right)}{\partial x_{1} \beta_{1}}-\frac{\partial\left(T_{\alpha_{1} \beta_{1}, \alpha_{2} \beta_{2}} X_{(1)}^{\alpha_{1}}\right)}{\partial x_{1}{ }^{\omega}}\right)=0 .
$$

If $\alpha_{1}^{\prime}=n+1, \alpha_{2}^{\prime}=n+1 ; \beta_{1}^{\prime}, \beta_{2}^{\prime} \neq n+1$, we have

$$
X_{(1)}^{\omega}\left(\frac{\partial\left(T_{\alpha_{1} \beta_{1}, \alpha_{2} \beta_{2}} X_{(1)}^{\alpha_{1}} X_{(2)}^{\alpha_{2}}\right)}{\partial x_{1}{ }^{\omega}}-\frac{\partial\left(T_{\alpha_{1} \omega, \alpha_{2} \beta_{2}} X_{(1)}^{\alpha_{1}} X_{(2)}^{\alpha_{2}}\right)}{\partial x_{1} \beta_{1}}\right)=0 .
$$

The reasoning which we have carried through for the case of the quadratic functional (12.7) of two-dimensional manifolds is directly extensible to a functional (12.4) of $r$-dimensional manifolds. We can therefore embody the results of this paragraph in the following important theorem.

TheOREM. A necessary and sufficient condition that the functional (12.6) of $r$-dimensional manifolds in $n+1$ dimensions be an invariant functional attached to the path curves of the one-parameter group (12.3) is that the functional (12.4) of $r$-dimensional manifolds in $n$ dimensions be a special* invariant functional of the one-parameter group

$$
\frac{d x^{i}}{d x^{n+1}}=x^{i} \quad(i=1,2, \cdots, n)
$$

with $x^{n+1}$ as the parameter of the group.

As a special case of the above theories we get obviously one of Cartan's $\dagger$ results which states that a complete integral invariant can always be formed from an integral invariant in the sense of Poincaré.

* See third footnote in $\$ 10$.

† Cf. his Leçons sur les Invariants Intégraux, pp. 28-29. 
13. Functionals admitting $s$-parameter groups. In Lie's theories of finite continuous groups an $s$-parameter group of transformations whose infinitesimal transformations have the symbols

$$
U^{(j)} f={ }^{(j)} X^{\omega}(M) \frac{\partial f}{\partial x^{\omega}} \quad(j=1,2, \cdots, s)
$$

satisfy the identities

$$
\left(U^{(\alpha)}, U^{(\beta)}\right) f=c_{\alpha \beta \gamma} U^{(\gamma)} f \quad(\alpha, \beta, \gamma=1,2, \cdots, s) .
$$

In (13.2), $\left(U^{(\alpha)}, U^{(\beta)}\right)$ is the alternant operator of Poisson and $c_{\alpha \beta \gamma}$ are the structural constants of the group.

A necessary and sufficient condition that a functional (5.4) of $r$-dimensional manifolds admit* the $s$-parameter group (13.1) is that it admit each one of the $s$ independent infinitesimal transformations of the group. Consequently with the aid of conditions (8.4) we see that the invariance conditions are

$$
\begin{aligned}
& \sum_{\lambda=1}^{i}\left[R_{\alpha_{1}}^{(\lambda)} \cdots \sigma_{1}, \cdots, \alpha_{i} \cdots \sigma_{i}\left(M_{1}, \cdots, M_{i}\right)\right. \\
& \left.+S_{\alpha_{1}}^{(\lambda)} \cdots \sigma_{1}, \cdots, \alpha_{i} \cdots \sigma_{i}\left(M_{1}, \cdots, M_{i}\right)\right]_{j}=0 \\
& \left(\alpha_{1}, \cdots, \sigma_{i}=1,2, \cdots, n ; j=1,2, \cdots, s\right) .
\end{aligned}
$$

In (13.3) the notation $\left[R_{\ldots}^{(\lambda)}+S_{\ldots}^{(\lambda)}\right]_{j}$ is used to denote the fact that ${ }^{(j)} X_{(k)}^{\omega}$ has been put in the place of $X_{(k)}^{\omega}$ in $\left[R_{\ldots}^{(\lambda)}+S_{\ldots}^{(\lambda)}\right]$. With this notation we can write immediately the necessary and sufficient conditions that a functional (5.4) of $r$-dimensional manifolds be an invariant functional attached $\dagger$ to the path curves of each one of the $s$ independent one-parameter groups defined by the $s$ independent infinitesimal transformations of the group (13.1). These conditions are

$$
\begin{aligned}
& {\left[R_{\alpha_{1}}^{(\lambda)} \cdots \sigma_{1}, \cdots, \alpha_{i} \cdots \sigma_{i}\left(M_{1}, \cdots, M_{i}\right)\right]_{j}=0} \\
& \quad\left[S_{\alpha_{1}} \cdots \rho_{1} \sigma_{1}, \cdots, \alpha_{k} \cdots p^{*}, \cdots, \alpha_{i} \cdots \rho_{i} \sigma_{i}\right. \\
& \left.\left(M_{1}, \cdots, M_{k}, \cdots, M_{i}\right)\right]_{j}=0 \\
& \left(\alpha_{1}, \cdots, \sigma_{i}=1,2, \cdots, n ; k, \lambda=1,2, \cdots, i ; j=1,2, \cdots, s\right) .
\end{aligned}
$$

There is one class of $s$-parameter groups for which we can affirm the existence of functional invariants corresponding to conditions (13.3) or to attached functionals corresponding to conditions (13.4). This is the case in

* This follows essentially from the familiar reasoning given in Lie's theories of invariant point functions.

$\dagger$ See $\$ 9$. 
which the structural constants $c_{\alpha \beta \gamma}$ of the group are all zero* and the number $s$ of essential parameters is at most equal to the number $n$ of dimensions. For such abelian groups, it is always possible* to find a coördinate transformation $(x)$ to $(y)$ such that in the $(y)$ coördinates the $s$ contravariant vectors ${ }^{(i)} X^{\omega}$ of the group have the components $\delta^{(i) \omega}$, where

$$
\begin{aligned}
\delta^{(i) \omega} & =0 \text { if } j \neq \omega \\
& =1 \text { if } j=\omega \quad(\omega=1,2, \cdots, n ; j=1,2, \cdots, s) .
\end{aligned}
$$

Observing therefore the conditions (13.3) and (13.4) in such a canonical set of coördinates $(y)$ we are enabled to state the following two interesting theorems.

THEOREM 1. There always exist functionals (5.4) of $r$-dimensional manifolds that admit an abelian $s$-parameter group $(s \leqq n)$; and for such a functional the components of the multiple tensor $T_{\alpha_{1}} \ldots \sigma_{1}, \ldots, \alpha_{i} \ldots \sigma_{i}$ in the canonical coordinate system $(y)$ are functions of the cogredient differences $y_{k}{ }^{j+1}-y_{k^{i}}$ $(j=1, \cdots, s-1 ; k=1,2, \cdots, i)$ and of the coördinates $y_{k}{ }^{l}(l=s+1, \cdots, n$; $k=1,2, \cdots, i)$.

THEOREM 2. There always exist invariant functionals (5.4) that are attached to the path curves of $s$ independent one-parameter groups of an abelian $s$-parameter group $(s \leqq n)$. The differential form $T(i) \delta x$ of such a functional, when expressed in the canonical variables $(y)$, depends only on the variables $y_{k}{ }^{l}(l=s+1, s+2, \cdots, n ; k=1,2, \cdots, i)$ and their differentials.

* Such s-parameter groups are called abelian groups. Cf. Bianchi, Lezioni sulla Teoria dei Gruppi Continui Finiti di Transformazioni, p. 260.

Princeton UntVersity, Princeton, N. J. 